\title{
Dihedral Angle Measurements for Structure Determination by Biomolecular Solid-State NMR Spectroscopy
}

\author{
Patrick C. A. van der Wel* \\ Solid-state NMR Group, Zernike Institute for Advanced Materials, University of Groningen, Groningen, Netherlands
}

In structural studies of immobilized, aggregated and self-assembled biomolecules, solid-state NMR (ssNMR) spectroscopy can provide valuable high-resolution structural information. Among the structural restraints provided by magic angle spinning (MAS) ssNMR the canonical focus is on inter-atomic distance measurements. In the current review, we examine the utility of ssNMR measurements of angular constraints, as a complement to distance-based structure determination. The focus is on direct measurements of angular restraints via the judicious recoupling of multiple anisotropic ssNMR parameters, such as dipolar couplings and chemical shift anisotropies. Recent applications are highlighted, with a focus on studies of nanocrystalline polypeptides, aggregated peptides and proteins, receptor-substrate interactions, and small molecule interactions with amyloid protein fibrils. The review also examines considerations of when and where ssNMR torsion angle experiments are (most) effective, and discusses challenges and opportunities for future applications.

Keywords: solid-state NMR, structural biology, amyloid, polyglutamine, protein structures

\section{INTRODUCTION}

In modern integrative structural biology, complementary structure determination methods provide insights into different aspects of the structure, dynamics and co-assembly of biomolecules. Tremendous advances in solid-state NMR (ssNMR) and in particular magicangle-spinning (MAS) NMR spectroscopy have widened the contribution of this technique to our understanding of protein aggregates and assemblies (Van der Wel, 2018). Descriptions of the process of structure determination via ssNMR spectroscopy often focus on the role of interatomic (or rather inter-nuclear) distances. However, NMR-based insights into the local geometry of protein backbones, in the form of dihedral angles, can also be a powerful tool in the NMR-based structural biology arsenal. In this review article we examine MAS ssNMR techniques that allow direct insights into angular constraints that define the structures of proteins and other biomolecules. Readers are also referred to earlier reviews in the mid 2000s (Hong, 2006; Hong and Wi, 2006; Ladizhansky, 2009), and the current review will focus on more recent applications since 2006. We shall also examine the place of these techniques in current and future biomolecular MAS ssNMR. Examples show how these restraints can be effective and even essential tools for structure determination of specific kinds of biological structures and assemblies. 

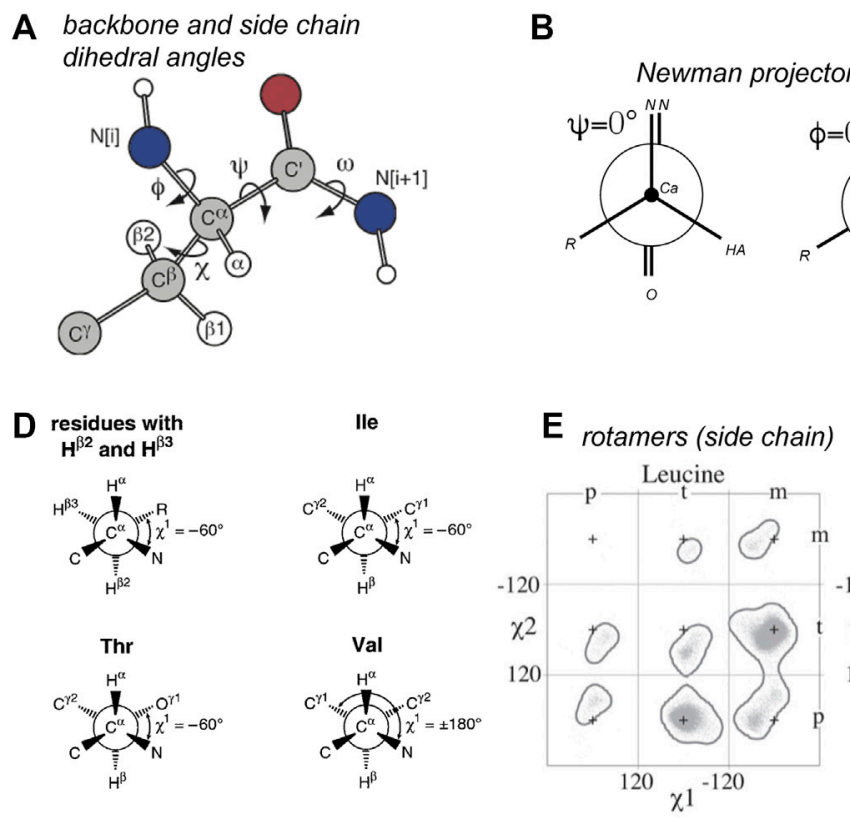

B

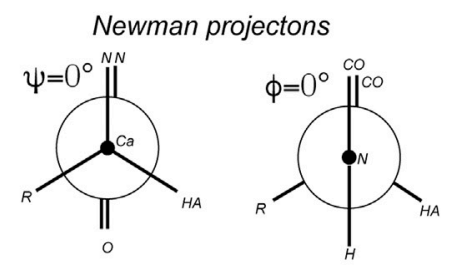

C

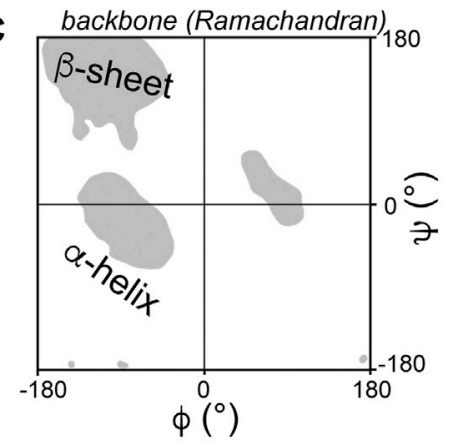

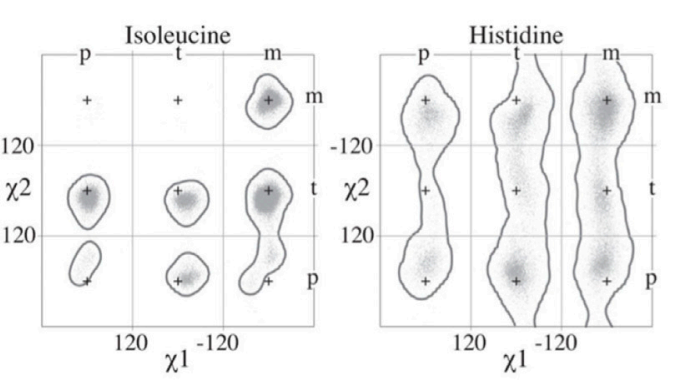

FIGURE 1 | Torsion angles in proteins. (A) Dihedral angles defining the protein backbone and side chain: $\phi$, $\psi$, and $\omega$, and $\chi$. (B) Newman projections of the $\phi$ and $\psi$ angles. The zero degree configuration of each is shown. (C) Ramachandran plot of $\phi$ and $\psi$ angles. Grey areas indicate the classic secondary structure regions that are most populated. Note that the $\phi=0^{\circ}$ from panel $B$ is not usually observed due to steric hindrance. (D) Definitions of the $\chi_{1}$ side chain angle. (E) Rotamer states for $\chi_{1}$ and $\chi_{2}$ for three residue types. Panel A is adapted with permission from (Hong et al., 1997a), (D) is adapted with permission from (Markley et al., 1998), and panel E from (Hintze et al., 2016).

\section{TORSION ANGLES IN PROTEINS}

By these dihedral or torsion angles we refer to the angle of two neighboring chemical bonds with each other (Figure 1). In protein structural biology the torsion angles that define the backbones of proteins are most commonly discussed, and they have a standard nomenclature (IUPAC-IUB Commission on Biochemical Nomenclature., 1970; Markley et al., 1998). These angles are defined and illustrated in Figures 1A,B. Among these the $\omega$ angle is not very variable, with it typically adopting a value of $\sim 180^{\circ}$ (trans). The cis configuration is rare, but when it occurs it can have notable biological consequences, for instance Pro cis/ trans isomerization is implicated in the aggregation process of the 及2-microglobulin protein (Torbeev and Hilvert, 2013; Mukhopadhyay et al., 2018). Of more common interest are the $\phi$ and $\psi$ backbone angles, which are visualized in Ramachandran plots (Figure 1C) (Ramachandran and Sasisekharan, 1968; Hovmöller et al., 2002). Only particular regions of Ramachandran space tend to be accessible, with the highly populated clusters representing secondary structure elements. The side chains of amino acids are also defined by named torsion angles, called $\chi_{1}, \chi_{2}$ etc., following an accepted nomenclature (Figures 1D,E) (IUPAC-IUB Commission on Biochemical Nomenclature., 1970; Markley et al., 1998; Lovell et al., 2000). These angles have preferred regions of geometric space, known as the preferred rotamer states that are nicely captured in rotamer libraries of evolving sophistication (Figures 1E,F) (Lovell et al., 2000; Dunbrack, 2002; Hintze et al., 2016).

\section{SSNMR TORSION ANGLE MEASUREMENTS-NCCN AS AN EXAMPLE}

\section{Technical Implementation and Analysis}

The general principle of torsion angle measurements by ssNMR is based on the measurement of the relative orientations of anisotropic ssNMR parameters, which in turn can be correlated to bond angles or orientations. The most straightforward and most common example involves the relative orientations of two different dipolar couplings, since these dipolar interactions are conveniently aligned with chemical bonds. For example, one can measure the relative orientation of the dipolar coupling vector (between ${ }^{13} \mathrm{C}$ and ${ }^{15} \mathrm{~N}$ ) of one C-N bond to the dipolar vector associated with a second $\mathrm{C}-\mathrm{N}$ bond. When these two vectors are associated with the $\mathrm{C} \alpha-\mathrm{N}$ and $\mathrm{C}^{\prime}$-N bonds in a polypeptide, the angle would be equivalent to the $\psi$ angle (Figure 1A). This is the approach behind the "NCCN" torsion angle experiments introduced in the late 1990s (Feng et al., 1997a; Costa et al., 1997).

The original implementation of NCCN measurements (Rienstra et al., 2002a, 2002b; Ladizhansky et al., 2003; Jaroniec et al., 2004; Bajaj et al., 2009; Barnes et al., 2009; van der Wel et al., 2010; Hoop et al., 2016) is as follows: a double quantum (DQ) state is generated between the directly bonded $\mathrm{Ca}$ and $\mathrm{C}^{\prime}$ carbons, e.g., via SPC5 dipolar recoupling (Figure 2A; red boxes). This DQ state is then submitted to ${ }^{15} \mathrm{~N}-{ }^{13} \mathrm{C}$ recoupling (commonly via the REDOR approach; Figure 2A, blue box). Since each carbon has a directly attached ${ }^{15} \mathrm{~N}$, they both 

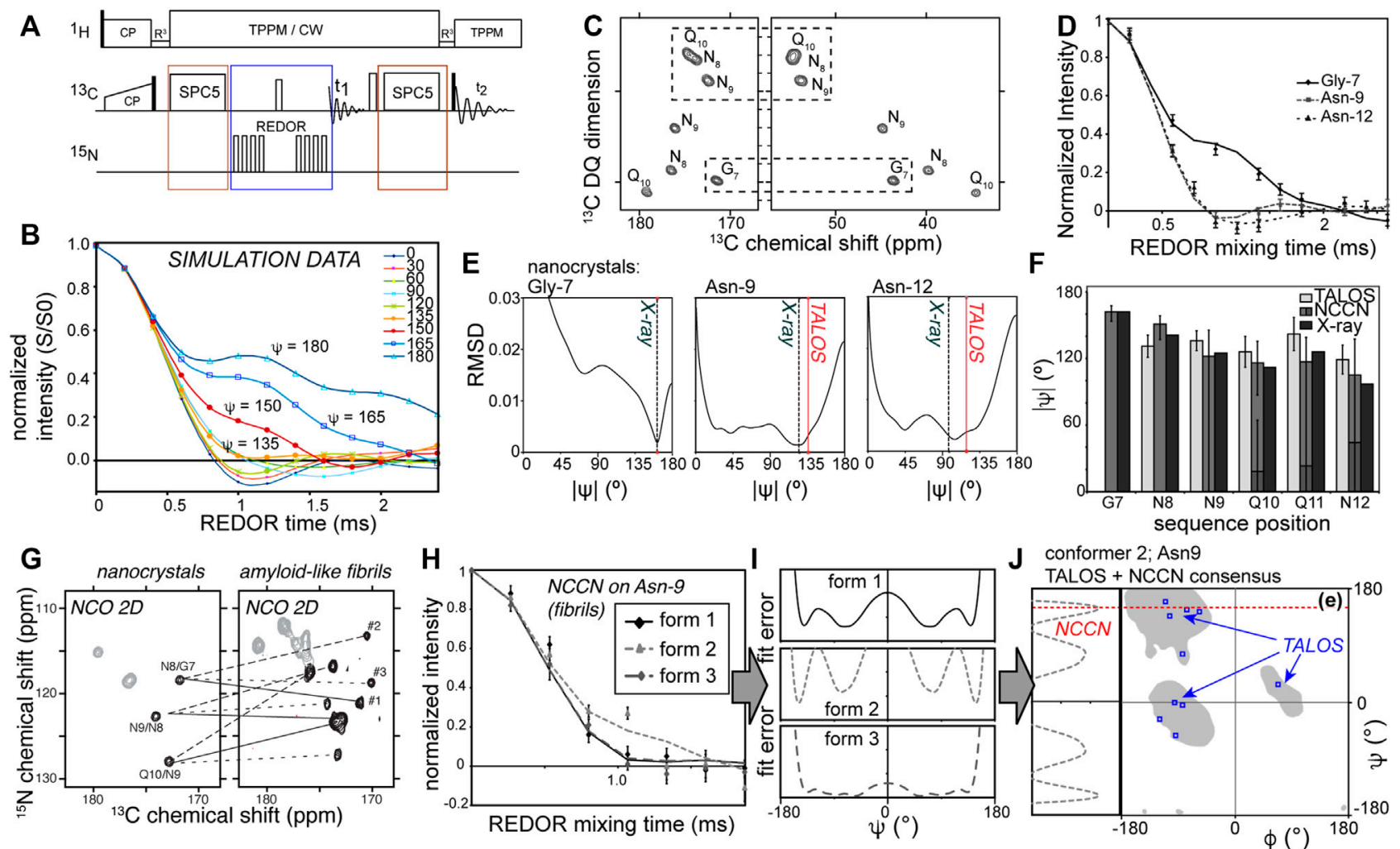

J conformer 2; Asn9

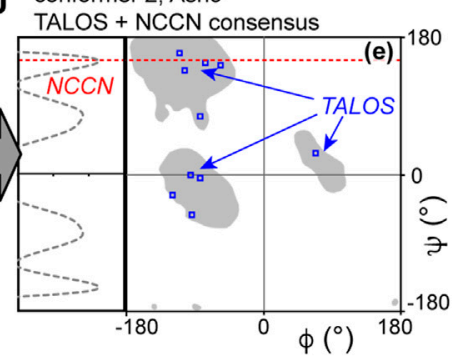

FIGURE 2 | Measuring backbone angle $\psi$ via NCCN experiments. (A) Pulse sequence for a $2 \mathrm{D}$ NCCN experiment, in which $2 \mathrm{D}{ }^{13} \mathrm{C}-{ }^{13} \mathrm{C}(\mathrm{SQ}-\mathrm{DQ}) \mathrm{spectra}$ are acquired for varying lengths of ${ }^{13} \mathrm{C}-{ }^{15} \mathrm{~N}$ dipolar mixing times. (B) Simulated NCCN dephasing curves, showing the S/SO intensity ratios as a function of the REDOR mix time. (C) Example 2D SQ-DQ spectrum acquired as part of a NCCN measurement of amyloid-like peptide nanocrystals, along with (D) experimental NCCN curves for three residues. (E) RMSD fit results of the experimental data, showing the minima. The X-ray and TALOS results are marked as well. (F) Bar graph of the NCCN best-fit results compared to the TALOS chemical shift analysis and the known X-ray structure data. The NCCN data match the X-ray data, with a better accuracy than TALOS analysis alone. (G) 2D NCO spectra for nanocrystalline and fibrillar GNNQQNY peptide (uniformly ${ }^{13} \mathrm{C}$, ${ }^{15} \mathrm{~N}$-labeled in the first four residues, marked as residues G7 through Q10 of the parent Sup35p protein). Lines show how each residue gives one signal in the crystals, but three peaks in the fibrils, which complicates distance measurements. (H) A NCCN experiment results probing the backbone angle $\psi$ for Asn-9 in the three co-existing fibril conformers. Two conformers have similar dephasing curves, but conformer \#2 diverges. (I) RMSD curves between simulation and experiment for the full range of $\psi$ angles, for each conformer. Note that the minima occur at multiple angles, and sometimes are broad, providing ambiguous constraints. (J) To resolve this one can combine chemical shift analysis (TALOS) with the NCCN constraints. In this example the 10 best TALOS "solutions" are not self-consistent, but by considering the NCCN angle, we pinpoint the optimal result in the $\beta$-sheet region near $\psi=150^{\circ}$. Panels C-J are reprinted with permission from ref. (van der Wel et al., 2010) copyright 2010 American Chemical Society.

experience rapid dephasing in a manner that is dominated by the directly-bonded ${ }^{15} \mathrm{~N}$. Whilst the orientation dependence of a single ${ }^{15} \mathrm{~N}-{ }^{13} \mathrm{C}$ REDOR recoupling experiment is masked in a typical MAS ssNMR study, these two ${ }^{15} \mathrm{~N}-{ }^{13} \mathrm{C}$ recoupling curves display an interference effect that results in behavior that is sensitive to the N-Ca-C'-N dihedral angle (Figure 2B).

Practically speaking, a typical REDOR approach is used to measure a relaxation-corrected dephasing curve (Figure $2 B$ ). A series of datasets with varying REDOR mixing times is acquired (keeping the DQ excitation time fixed). The relaxation correction involves the measurement (at each REDOR time) for each peak of interest of a "S" signal (with active ${ }^{15} \mathrm{~N}$ REDOR pulses) and "S0" signal (without ${ }^{15} \mathrm{~N}$ REDOR pulses). The $\mathrm{S} / \mathrm{S} 0$ peak intensity ratio is then plotted, yielding angle-dependent curves as shown in Figure 2B. At each time point either a $1 \mathrm{D}$ or $2 \mathrm{D}$ spectrum is acquired, with the 2D implementation shown in Figures 2A,C (Ladizhansky et al., 2003; van der Wel et al., 2010). The main benefit of the $2 \mathrm{D}$ version is that it allows one to resolve many signals at once (Ladizhansky et al., 2003). Naturally, it comes at the expense of signal-to-noise (per unit time), which is superior in the $1 \mathrm{D}$ versions. It is worth noting that the signal-to-noise can be a challenge in these experiments. This stems from the fact that a clear distinction of the different dihedral angles only occurs in those time points where already extensive dephasing has occurred (i.e., much of the signal is lost; see Figure 2B). Moreover, in this region, the differences between some of the dihedral angles can be quite small, such that a very good signal to noise may be required to narrow down a specific angle. Achieving a good signal-to-noise level is inherently a challenge due to the polarization losses associated with the multiple recoupling steps (both the ${ }^{13} \mathrm{C}$ DQ recoupling and filtering, and also the heteronuclear recoupling), both due to losses inherent in the DQ filtering and relaxation processes.

Analysis and interpretation of the obtained data is done by comparing experimental data points (i.e., the $\mathrm{S} / \mathrm{S} 0$ ratios) to reference curves such as the data in Figure $\mathbf{2 B}$. The latter can 


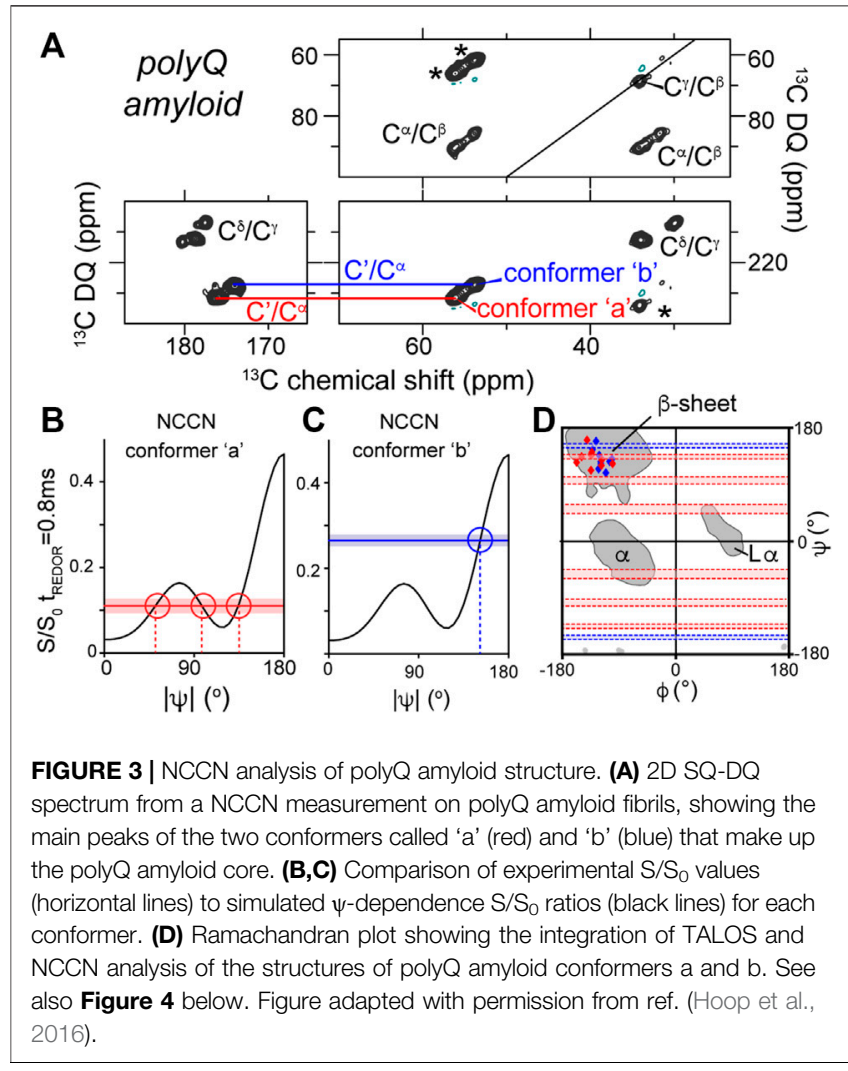

be derived from analytical descriptions of the experiment. In our own analyses we typically employ primarily numerical simulations to generate the curves. A relevant spin system is modelled for the range of candidate dihedral angles (or molecular structures), resulting in a library of reference curves. For some types of torsion angle experiments it may also be necessary to incorporate in this "library" relaxation effects, the impact of the chemical shift anisotropy (CSA), and dynamic modulation (van der Wel et al., 2009; Hou et al., 2010; Hoop et al., 2014). Naturally, these additional free parameters increase the complexity of the analysis and reduce the expected precision of obtained results.

Additionally, it is worth noting that a dipolar recoupling experiment like REDOR reflects a through-space interaction and can therefore invoke multispin interactions in extensively or fully isotope-labeled samples. Such multispin interactions would not invalidate the dihedral angle experiment, as it is essentially based on seeing a dependence of the observed signal on the dihedral angle in question. This dependency would be modulated, but not be negated by multispin interactions, unless non-local interactions would dominate over the local interactions which are supposed to be recoupled. However, in this case the effect of "dipolar truncation", in which strong dipolar interactions suppress or truncate the contributions from longer range interactions (Bayro et al., 2009), is actually beneficial. Directly-bonded ${ }^{13} \mathrm{C}-{ }^{15} \mathrm{~N}$ interactions will effectively truncate the effect of long-range inter-residue and inter-molecular interactions. It is also worth noting that we benefit here from the relatively low density of ${ }^{15} \mathrm{~N}$ sites in polypeptides, which allows for the trains of ${ }^{15} \mathrm{~N}$ REDOR pulses without detrimental ${ }^{15} \mathrm{~N}-{ }^{15} \mathrm{~N}$ recoupling (illustrated in Figure 2A). Another important observation is that these experiments can be applied to polypeptides outfitted with uniform ${ }^{13} \mathrm{C}$ and ${ }^{15} \mathrm{~N}$ labeling, without need for site- or residue-specific isotope labeling as may be needed for certain other types of torsion angle experiments. The examples in Figures 2, 3 are obtained with synthetic peptides where short stretches of residues were labeled, but the same experiments can be applied to fully labeled proteins (Ladizhansky et al., 2003).

\section{Example Application to Nanocrystalline Peptides With Known Structures}

The data in Figures 2C-F represent validation experiments in an amyloid-like, but nanocrystalline, peptide assembly. Integrated peak volumes from the SQ-DQ 2D spectrum (Figure 2C) are plotted as $\mathrm{S} / \mathrm{S} 0$ ratios as a function of the REDOR recoupling time. The example curves in Figure 2D show the variable differences between residues, with some of the angles hard to distinguish (see also Figure 2B for $\psi<130^{\circ}$ ). Figure 2E shows the results of fitting the experimental curves, yielding in some cases multiple minima. This illustrates one common challenge of torsion angle experiments, which is that they often have regions of angular space where the obtained dephasing curves are essentially indistinguishable. As reviewed in earlier work (Hong, 2006; Hong and Wi, 2006; Ladizhansky, 2009), this issue can be addressed by combining multiple types of dihedral angle measurements with complementary sensitivities. Notably, the NCCN experiment minima are close to both the $\mathrm{X}$-ray structure angles and the results of NCCN-based chemical shift analysis. This is illustrated in the bar graphs of Figure 2F. These results show that the NCCN experiment gives results that match the structure as known from X-ray microcrystallography (Nelson et al., 2005; van der Wel et al., 2007, 2010), consistent with earlier studies (Ladizhansky et al., 2003).

\section{Studies of Unknown Structures in Amyloid Fibrils}

Several studies have deployed NCCN experiments to study unknown structures of amyloid-like fibrils (Jaroniec et al., 2004; van der Wel et al., 2010; Hoop et al., 2016). For the fibrils formed by the abovementioned prion-derived model peptide GNNQQNY, these experiments detected the anticipated $\beta$-sheet structures typical of amyloids, but also non- $\beta$ structure as an integral part of a composite fibril structure (van der Wel et al., 2010; Lewandowski et al., 2011). Notably this feature was present in one of three conformers (monomer structures) that composed the "composite" fiber architecture (which manifest as three peaks per residue; Figure 2G). This feature made the reliance on distance measurements more difficult, due to substantial peak overlap. Thus, the complementary torsion angle measurements were particularly helpful and valuable. The three conformers' distinct structures are clear in the torsion angle data, as illustrated for residue Asn-9 in Figure 2H. The presence of a 
non- $\beta$ kink or turn was at first surprising, as it is absent from the nanocrystals. However, nowadays this finding is reminiscent of the common presence of kinks, turns or bends in amyloid architectures (between quite short $\beta$-strand segments) (van der Wel, 2017; Sawaya et al., 2021). Figures 2I,J shows how the experimental ssNMR data were translated to dihedral angles. The fit between the experimental data and simulated curves was evaluated as a function of the simulated dihedral angle (Figure 2I). In some cases this shows consistency (i.e., low RMSD between the experimental data and simulated data) with multiple possible angles. One approach to overcome this ambiguity, shown in Figure 2J, is to combine the optimal NCCN angles with TALOS-based chemical shift analysis, in order to obtain a unique solution not accessible through either approach alone.

A similar structural complexity was subsequently detected in ssNMR studies of polyglutamine (polyQ) amyloid structure, which was found to consistently contain two distinct types of peptide conformations (Figure 3A) (Schneider et al., 2011; Sivanandam et al., 2011; Hoop et al., 2014, Hoop et al., 2016; Isas et al., 2015; Matlahov and van der Wel, 2019). Proteins with expanded polyQ domains are of biomedical interest as they represent the molecular basis of a series of CAG repeat expansion disorders, which remain as-yet incurable and untreatable (Wetzel, 2012). The disease-associated mutant proteins are prone to aggregation and form toxic aggregates, which include fibrillar structures with all the hallmarks of typical amyloid fibrils. Unlike several other amyloid proteins (van der Wel, 2017; Sawaya et al., 2021), the 3D atomic structure of none of the pathogenic polyQ protein aggregates is as yet known (Matlahov and van der Wel, 2019). The degenerate nature of the primary sequence of these polypeptides greatly complicates structural analysis by ssNMR, which has made the deployment of torsion angle measurements valuable and indeed essential. Structural study by distance measurements is further complicated by the already noted composite nature of the amyloid core structure, presenting as two peaks for each residue (marked "a" and "b" in Figure 3). By combining NCCN measurements and TALOS-based chemical shift analysis, it was shown that these two conformers reflect two types of $\beta$-strand structures with distinct backbone conformations (Figures 3A-D) (Hoop et al., 2016). The origin of this characteristic doubled-peaks signature stems from the presence of antiparallel $\beta$-sheets in these fibrils, which contain $\beta$-hairpin motifs for the longer polyQ expansion lengths (more about polyQ structures below) (Matlahov and van der Wel, 2019).

\section{BEYOND THE PROTEIN BACKBONE-HCCH EXPERIMENTS}

The first examples of direct MAS ssNMR torsion angle measurements were the HCCH experiments (Feng et al., 1996; Feng et al., 1997b), whose principles were also discussed in some detail in prior reviews (Hong and Wi, 2006; Ladizhansky, 2009). Briefly, again a DQ state is generated between two directly bonded ${ }^{13} \mathrm{C}$ carbons, but now it is combined with controlled recoupling of the $\mathrm{C}-\mathrm{H}$ dipolar interaction (Figure 4A). Keeping the DQ excitation time fixed, the $\mathrm{C}-\mathrm{H}$ recoupling time is varied. This yields a time-dependent decrease in the DQ signal, with the shape of the curve dependent on the geometry (i.e., torsion angle) of the HC-CH spin system (Figure 4B) (Feng et al., 1997b; Bajaj et al., 2009; Edwards et al., 2010; Hoop et al., 2016). Whilst initial applications focused on organic molecules and retinal structures, recent applications have used this technique to probe the side chain dihedral angles of amino acids (Rienstra et al., 2002b; Bajaj et al., 2009; Edwards et al., 2010; Hoop et al., 2016). Like the NCCN experiment, these $\mathrm{HCCH}$ measurements can be applied to uniformly ${ }^{13} \mathrm{C}$ labeled residues and polypeptides. In contrast to backbone torsion angles, the side chain angles are (at this time) not accessible via the kind of chemical shift analysis applied to polypeptide backbones (e.g., TALOS; Figure 2).

\section{Polyglutamine Amyloid Steric Zippers}

One recent application of the $\mathrm{HCCH}$ experiment was also in our own work on aggregated polyglutamine (polyQ) proteins. As noted above, these protein aggregates are hard to study by distance measurements alone, requiring the application of dihedral angle measurements for structure determination. The glutamine residues feature an extended aliphatic side chain, with two methylene $\left(\mathrm{CH}_{2}\right)$ groups for the $\mathrm{C} \beta$ and $\mathrm{C} \gamma$ atoms. This permitted the use of $\mathrm{HC}-\mathrm{CH}$ dihedral angle measurements of the $\chi_{1}$ and $\chi_{2}$ angles of these amino acids. Figure 4B shows how the $\chi_{2}$ angles of 60 and $180^{\circ}$ give clearly different $\mathrm{HCCH}$ dephasing curves, with the dephasing at the mid-point (1/2 rotor period) varying with the $\chi_{2}$ angle (Figure $4 \mathrm{C}$ ). For a practical experiment, measuring up to this mid-point is sufficient, as shown in Figures 4D,E. These results identified the $\chi_{2}$ angle for the glutamine residues in polyQ amyloid to be near $180^{\circ}$, in contradiction to certain prior structural models based on (low-resolution) X-ray diffraction (Sharma et al., 2005; Hoop et al., 2016). Similarly, the same experiments can be used to probe the $\chi_{1}$ angle (Figure $4 F$ ), adding further structural constraints on the polyQ amyloid core structure (Figure 4G). As summarized in Figure $\mathbf{4 H}$, one unfortunate aspect of the $\chi_{1}$ measurements is that they were able to exclude various conformations, but did not result in a completely unambiguous single angle value. As noted above, this is not uncommon for dihedral angle measurements, which can provide a unique solution in some cases $\left(\chi_{2} \sim 180^{\circ}\right)$ but only partially constrain the angle in other cases. The model in Figure 4G represents a visualization of the best-fit backbone and side chain angle results. This model shows how the two thusobtained conformers ( $\beta$-strand types a and b) are conformationally compatible, and explain the co-assembly of the polyQ amyloid core. Notably, prior structural models derived from other techniques were not consistent with the obtained ssNMR results (Hoop et al., 2016).

\section{Receptor-Substrate Interactions}

Another notable use of the $\mathrm{HCCH}$ experiments is a nice study of a small molecule substrate (glutamate) bound to a receptor protein, which predated our work on polyQ (Edwards et al., 2010). An isotopically labeled substrate was bound to the ionotropic glutamate receptor 2, which was itself unlabeled (Figure 5). 


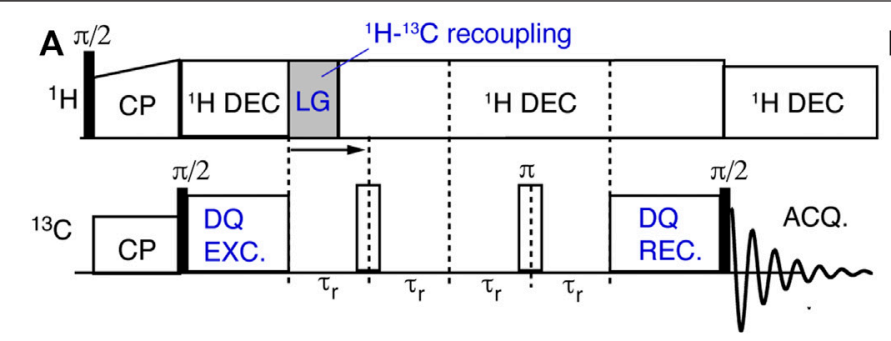

D

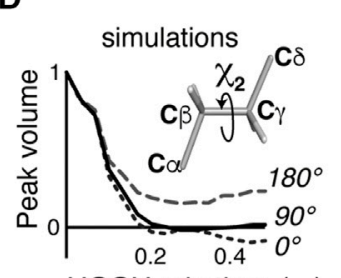

E

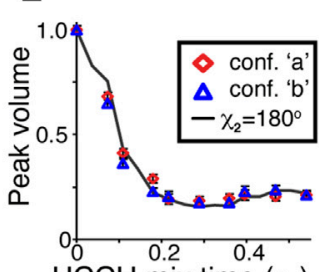

F

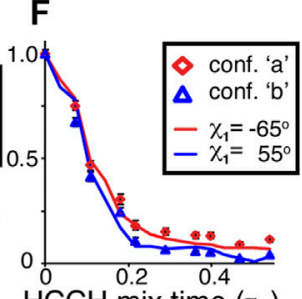

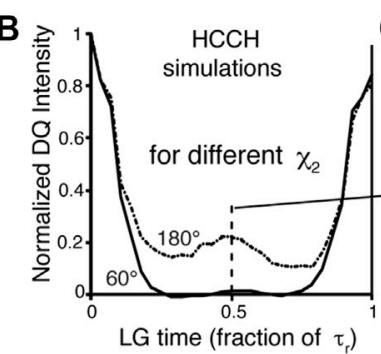

G

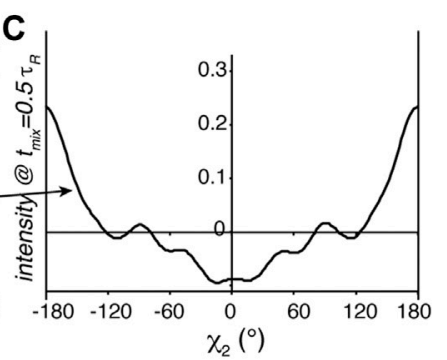

$\mathrm{HCCH}$ mix time $\left(\tau_{\mathrm{R}}\right)$

H
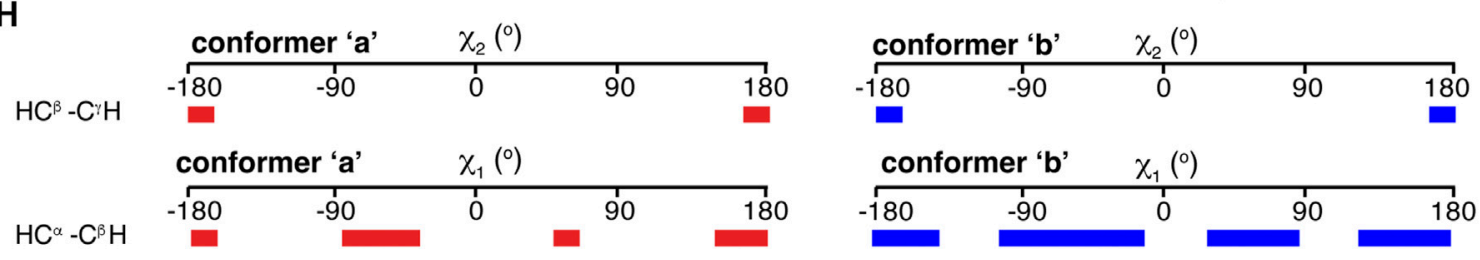

FIGURE 4 | $\mathrm{HCCH}$ experiments for measuring side chain angles. (A) Pulse program schematic for a HCCH experiment. (B) Simulated HCCH curves for measurement of the $\chi_{2}$ angle in glutamine residues $\left(\mathrm{a} \mathrm{C} \beta \mathrm{H}_{2}-\mathrm{C} \gamma \mathrm{H}_{2}\right.$ spin system), for $\chi_{2}=60$ or $180^{\circ}$. (C) Variation of the mid-rotor-period intensity as a function of the $\chi_{2}$ angle. (D,E) Simulated and experimental data measuring the $\chi_{2}$ angle of glutamine residues in the core of polyglutamine amyloid. (F) Application to the $\chi_{1}$ angle in polyglutamine amyloid. (G) Visualized structural model for how the polyglutamine amyloid core is structured, based on the best-fit dihedral angle solutions. (H) Overview of the ambiguity in some, but not all, of the measured $\chi$ angles. The colored bars indicate those angles that are consistent with the observed torsion angle curves. The red bars on the left apply to conformer 'a', and the blue ones (right) to conformer 'b'. Figure adapted from ref. (Hoop et al., 2016) with permission.
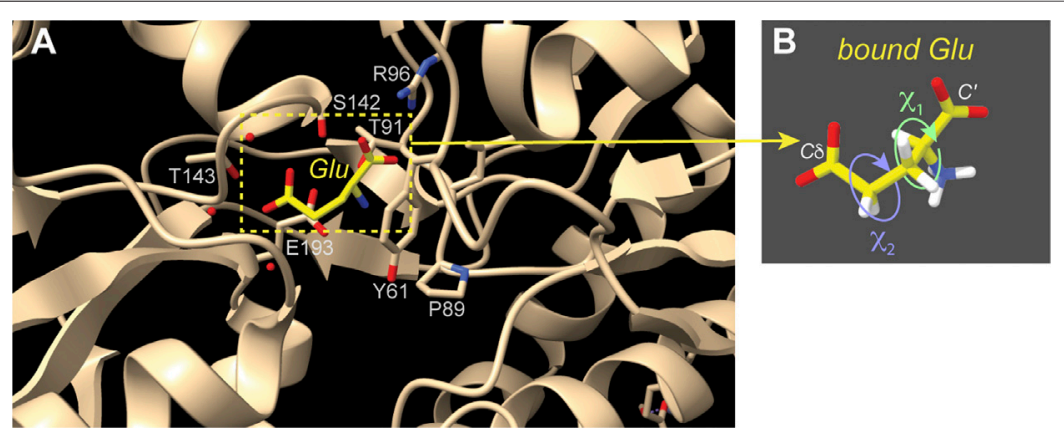

FIGURE 5| Receptor-bound substrate studied by torsion angle ssNMR measurements. (A) This image shows the structure of the receptor-bound Glu amino acid, based on X-ray crystallography (PDB 1FTJ) (Armstrong and Gouaux, 2000). Selected residues surrounding the bound substrate are indicated. (B) HCCH ssNMR analysis of $\chi_{1}$ and $\chi_{2}$ side chain angles enabled the measurement of the Glu residue structure while it was bound to the crystalline unlabeled protein (Edwards et al., 2010 ). This figure was prepared with UCSF ChimeraX (Pettersen et al., 2021).

Two torsion angles, defining $\chi_{1}$ and $\chi_{2}$ of the Glu (Figure 5B), were measured with $\mathrm{HCCH}$-type experiments. When combined with REDOR-based distance constraints, these data defined the conformation of the receptor-bound substrate. The reliability of the method was validated by comparison to known crystal structures (Figure 5A), with the proof-of-principle ssNMR study being applied to the crystalline receptor-substrate complex, in which the glutamate substrate is uniformly labeled. The $\mathrm{HCCH}$ measurements represented analogous experiments to our own subsequent studies of the polyQ amyloid structure (Figure 4). The individual dihedral angle measurements were again consistent with multiple distinct 


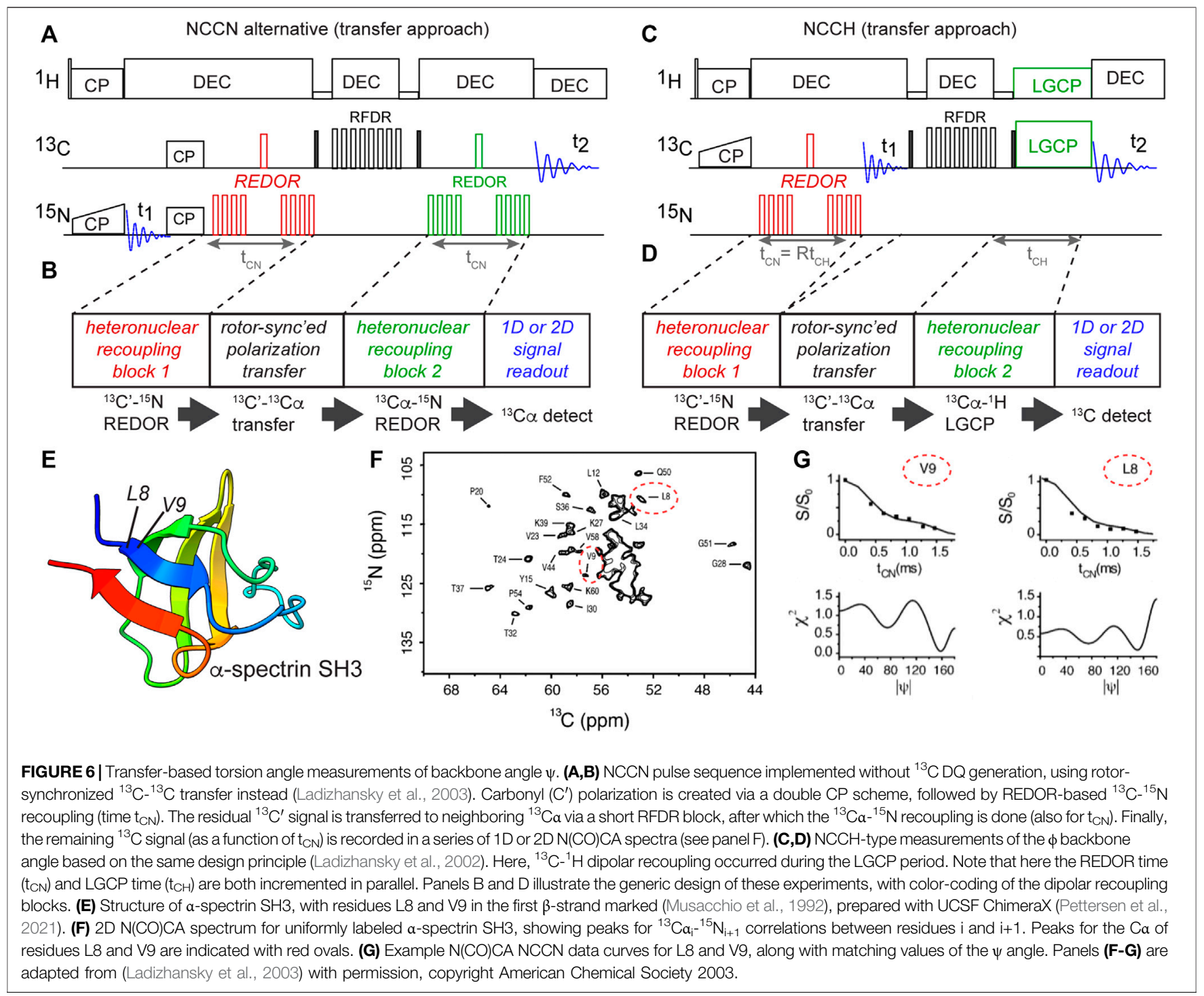

solutions, but by combining the different data with a REDORbased distance measurement, a unique structural solution was obtained. The obtained conformation matched X-ray based structures previously determined, as illustrated in Figure $\mathbf{5}$ (Armstrong and Gouaux, 2000).

\section{BACK TO THE BACKBONE-ALTERNATIVE IMPLEMENTATIONS}

In our hands, the abovementioned DQ-based approach is particularly powerful and robust. However, also alternative implementations of analogous torsion angle measurements have been demonstrated. This is illustrated via a different implementation of the NCCN-type measurement (Figures 6A,B) (Ladizhansky et al., 2003). This experiment follows the general approach shown in panel B: having two distinct recoupling blocks sandwiching a polarization transfer block. A key aspect of the pulse sequence is that each of these blocks should ideally be deployed in a rotor-synchronized (and/or constant-time) manner, such that individual molecules (crystallites) are recoupled at the same orientation relative to the magnetic field. This method has the benefit that it can be quite flexibly deployed, for example in the form of the HCCN experiment shown in Figures 6C,D (Ladizhansky et al., 2002). This HCCN experiment can be used to determine the $\psi$ backbone angle, especially in $\alpha$-helical secondary structures, thus complementing the NCCN experiment in terms of its applicability.

An illustration of the application of the abovementioned NCCN experiment from Figure 6A is shown in Figures 6E,F, from a study on crystalline uniformly ${ }^{13} \mathrm{C},{ }^{15} \mathrm{~N}$-labeled $\alpha$-spectrin protein (Figure 6E) (Ladizhansky et al., 2003). Integrating peaks in a series of $2 \mathrm{D} \mathrm{N}(\mathrm{CO}) \mathrm{CA}$ spectra (Figure 6F), the REDOR dephasing curves for individual residues were measured. The $\beta$-sheet residues 8 and 9 are marked in the spectrum, with their 

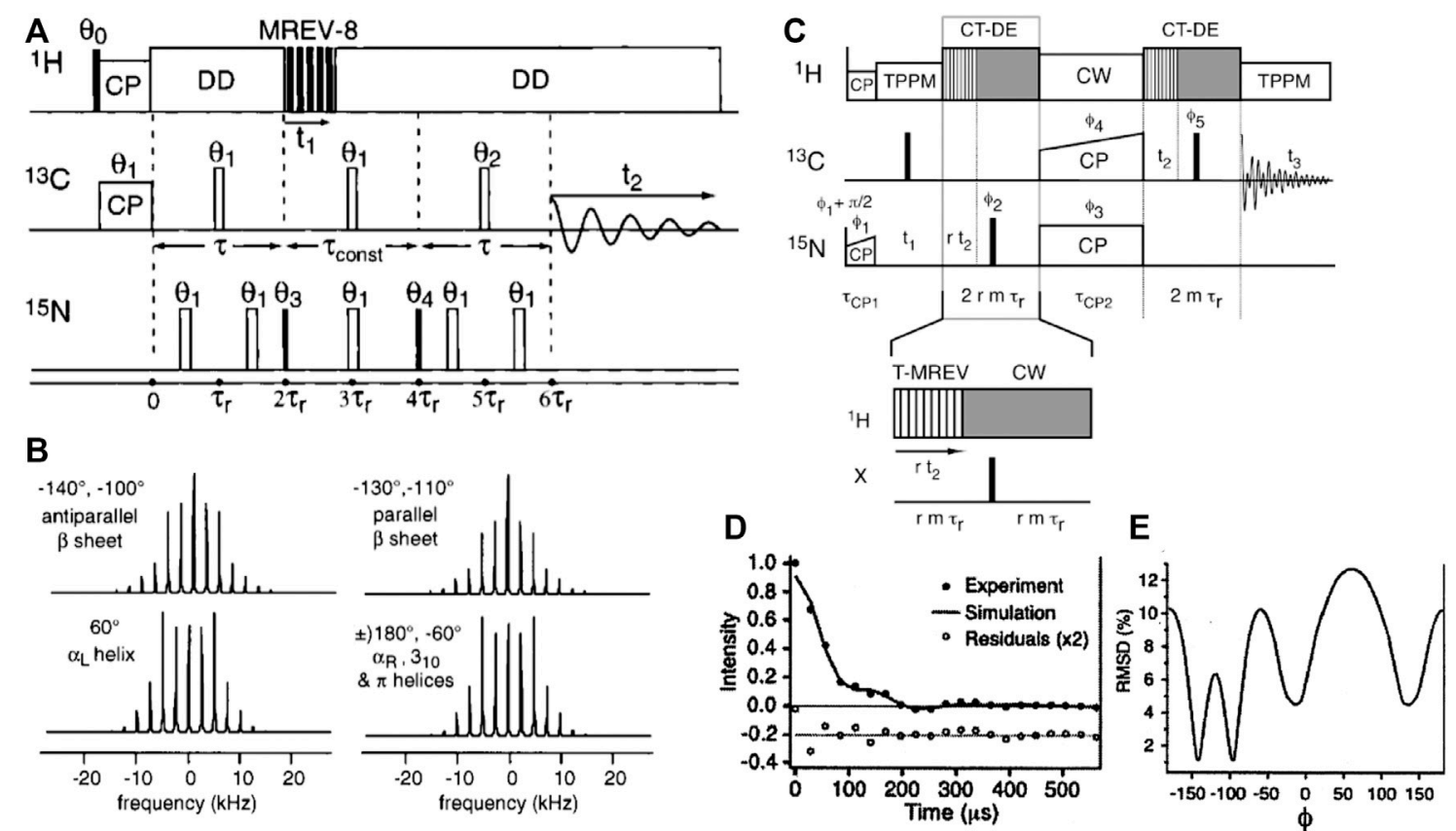

FIGURE 7 | HCNH measurement of the $\phi$ backbone angle. (A) Pulse sequence for the $\mathrm{HCNH}$ experiment, where $\mathrm{MREV}-8$ is used to achieve ${ }^{1} \mathrm{H}-{ }^{13} \mathrm{C} /{ }^{15} \mathrm{~N}$ dipolar recoupling of ${ }^{13} \mathrm{C}-{ }^{15} \mathrm{~N}$ correlations. (B) Example dipolar lineshapes for four different $\phi$ angles, based on simulations. Note that one lineshape often represents two distinct $\phi$ angles, as indicated. The figure also lists the matching secondary structures, showing that each type of secondary structure has a characteristic spectral lineshape. (C) Pulse sequence of a HCNH experiment based on a transfer-like implementation similar to those shown in Figure 6. (D,E) Representative results showing how also this variant points to multiple minima. Adapted with permission from (A,B) ref. (Hong et al., 1997a) and (C-E) (Rienstra et al., $2002 a)$, Copyright 1997 and 2002 American Chemical Society.

NCCN curves shown in Figure 6G. Once again, multiple minima can be observed, e.g. for residue L8.

To measure the $\phi$ backbone angle one can use the $\mathrm{HNCH}$ ssNMR experiment (Hong et al., 1997a, Hong et al., 1997b; Hong, 1999; Rienstra et al., 2002a). Also this experiment can be implemented in different ways (Figure 7), either via coherence generation or a rotor-synchronized transfer approach (Rienstra et al., 2002a). The corresponding pulse sequences are illustrated in Figure 7, along with selected results from the literature. Once more these data illustrate how a single torsion angle measurement commonly is consistent with multiple best-fit minima. As noted above, this ambiguity can be resolved by performing multiple different torsion angle measurements, integration with chemicalshift-based analysis, consideration of accessible Ramachandran/ rotamer space, and the measurement of relevant distance measurements. The reader is referred to prior review articles for a more in-depth discussion of these techniques (Hong and $\mathrm{Wi}$, 2006; Ladizhansky, 2009).

\section{USING CSA TENSORS FOR MEASURING $\omega$ AND MORE}

The most intuitive types of dihedral angle measurements are arguably those in which one recouples dipolar interactions that align nicely with chemical bonds, as discussed above. However, also the anisotropy of the chemical shift (CSA) can be deployed to good effect, either by recoupling two CSAs to each other, or by combining CSA recoupling with dipolar recoupling. Examples of these approaches were also introduced in the late 1990s (Ishii et al., 1996; Weliky and Tycko, 1996; Bower et al., 1999). The experiment in its simplest form involved simply slow MAS along with ${ }^{13} \mathrm{C}$ ${ }^{13} \mathrm{C}$ mixing, but this depended on ${ }^{13} \mathrm{CO}$-only labeling (Weliky and Tycko, 1996). It would not work in this form for a uniformly ${ }^{13} \mathrm{C}$ labeled sample. An interesting recent application of this experiment was used to examine the conformation of the amyloid-binding fluorescent dye congo red, in its fibril-bound state (Figure 8). The CSAbased torsion angle measurement determined the central bond in the amyloid-bound dye, complementing other structural studies on the dye-fibril interactions by the same research groups (Schütz et al., 2011; Gowda et al., 2017). In this case, site-selective ${ }^{13} \mathrm{C}$ labeling of the congo red was used to label two aromatic carbons. These aromatic sites have large ${ }^{13} \mathrm{C}$ CSAs, with a well-understood orientation of the CSA tensor relative to the molecular frame. Thus, by determining the relative orientations of the two CSA tensors, one can determine the rotational angle marked in Figure 8A. Free rotation around this bond causes the unbound dye to display low fluorescence. Upon binding to amyloid fiber surfaces, the immobilization of the dye boost the fluorescence and permits thereby the selective detection of amyloid-like structures (Schütz et al., 2011; Yakupova et al., 2019). The 


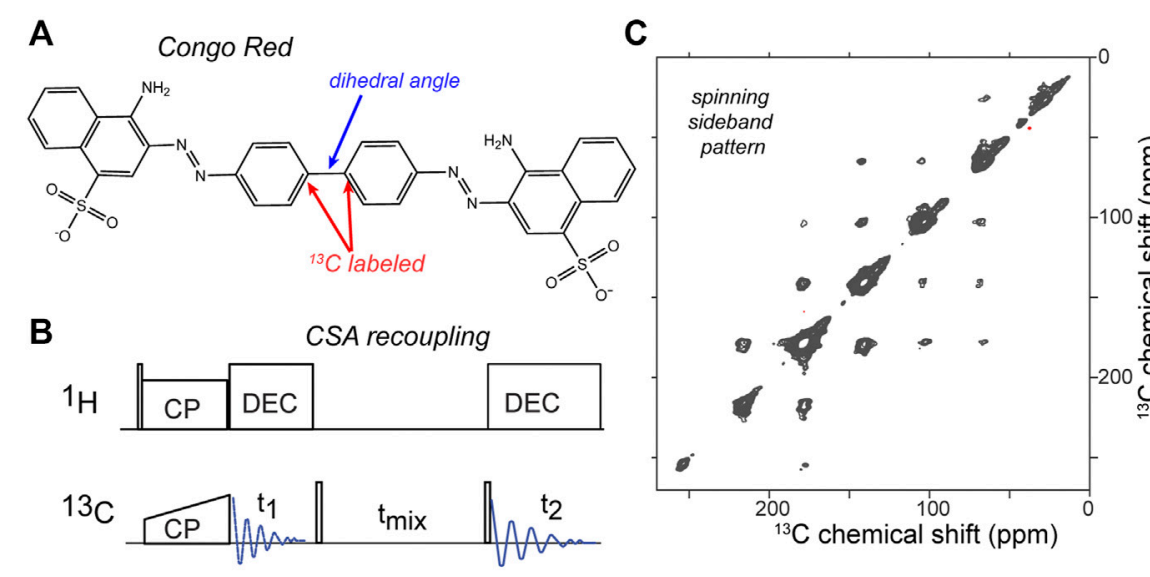

D Amyloid-bound Congo Red (2011)

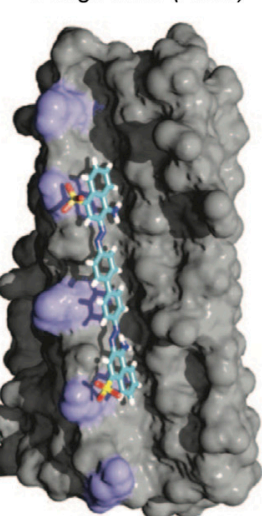

FIGURE 8 | CSA-recoupling with selective labeling: amyloid dye Congo Red. (A) Chemical structure of congo red, which is widely used to detect amyloid structures. For the CSA recoupling experiments the 1 and $1^{\prime}$ carbons are ${ }^{13} \mathrm{C}$ labelled (marked red), in order to measure the marked dihedral angle (blue). (B) Pulse sequence used for the CSA recoupling experiment used by (Gowda et al., 2017) to measure the dihedral angle marked in (A). (C) $2 \mathrm{D}{ }^{13} \mathrm{C}-{ }^{13} \mathrm{C}$ tensor correlation spectrum at $8 \mathrm{kHz}$ MAS on a $850-\mathrm{MHz}\left({ }^{1} \mathrm{H}\right)$ spectrometer, using the indicated pulse sequence with a 50 -ms rotor-synchronized ${ }^{13} \mathrm{C}-{ }^{13} \mathrm{C}$ transfer time. The sample contained HET-s amyloid fibrils with the selectively labeled congo red bound. For more details see ref. (Gowda et al., 2017). (D) Structural model of amyloid-bound congo red from prior ssNMR studies by the same groups, adapted with permission from ref. (Schütz et al., 2011).

A

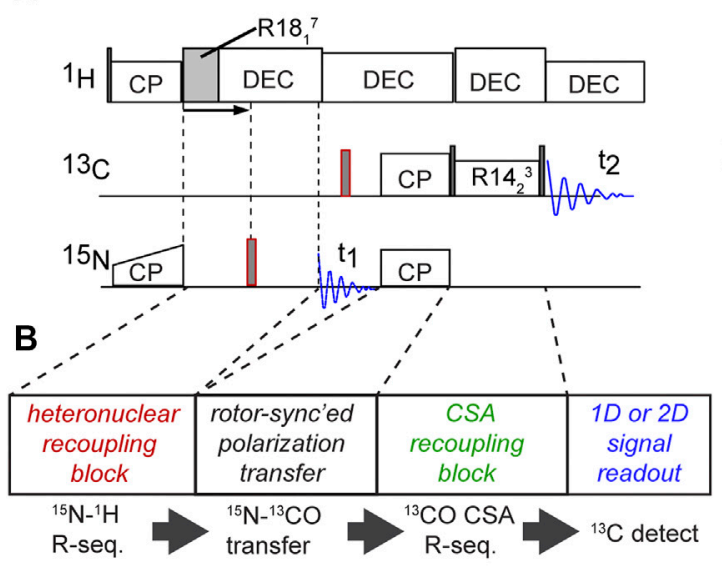

C<smiles>CCCCC(NCCCN)C(=O)O</smiles><smiles>O=C1CC2CNC(CN2)C1</smiles>

DKP

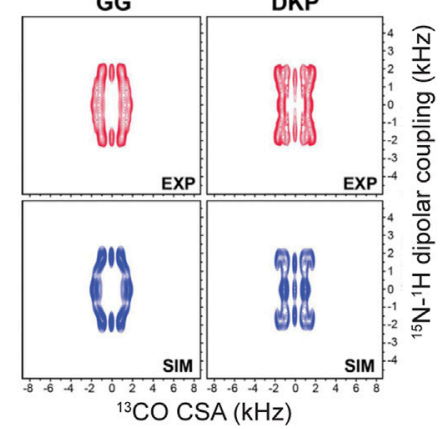

FIGURE 9 | Hybrid CSA/dipolar recoupling experiment. (A) Pulse sequence and (B) its building blocks. (C) Chemical structures of test molecules with different molecular configurations mimicking the cis or trans peptide bond angle $\omega$. (D) 2D CSA-dipolar correlation spectra for the two reference compounds representing the distinct $\omega$ angles. Panel (D) was adapted with permission from ref. (Mukhopadhyay et al., 2018).

experiment was performed with a fairly straightforward pulse sequence (Figure 8B), that allowed the measurement of a $2 \mathrm{D}$ ${ }^{13} \mathrm{C}-{ }^{13} \mathrm{C}$ spectrum that at slow MAS rates (here $8 \mathrm{kHz}$ ) showed a spinning side band pattern as illustrated in Figure 8C. Analysis of the peak pattern allowed the determination of the abovementioned torsion angle, which could then be used to refine the structural model of the congo red bound to the fiber surface grooves (Figure 8D). This represents a nice example of how one can target a torsion angle measurement to a particular (biological) question.

Moreover, hybrid methods can also determine the relative orientations of a CSA tensor and a dipolar coupling (Ishii et al.,
1996; Fujiwara et al., 1997; Hong et al., 1998; Chan and Tycko, 2003; Hou et al., 2010; Mukhopadhyay et al., 2018). In these MAS ssNMR experiments, different kinds of pulse sequence elements are used to actively recouple the CSA under medium/fast MAS $(>10 \mathrm{kHz})$, unlike the slow-MAS $(<10 \mathrm{kHz})$ CSA measurement mentioned above. For example, recent studies have used either ROCSA and R-sequence-based CSA recoupling techniques (Chan and Tycko, 2003; Hou et al., 2010; Mukhopadhyay et al., 2018). This approach also makes it feasible to apply these experiments without selective labeling strategies, enabling studies of fully ${ }^{13} \mathrm{C}$-labeled proteins. Figure 9 shows a recent example in which R-sequences were used for both CSA 
recoupling and dipolar recoupling, in order to enable measurement of the peptide bond angle $\omega$ (Mukhopadhyay et al., 2018). The figure shows the implementation and results for two model compounds (Figure 9C), but the original paper includes application to crystalline and fibrillar protein samples as well. This experiment and figure also illustrate a few relevant concepts, common to dihedral angle measurements. Figure 9D shows a $2 \mathrm{D}$ spectrum in which none of the axes show the (isotropic) chemical shift, but rather the anisotropic parameters: the ${ }^{13} \mathrm{C} \mathrm{CSA}$ and the ${ }^{1} \mathrm{H}-{ }^{15} \mathrm{~N}$ dipolar coupling strength. Prior work has proposed the term Relayed Anisotropy Correlation spectra (RACO) (Ishii et al., 1996). In this experiment, these CSA and dipolar recoupling periods are both independently incremented, resulting in these typical 2D data. This figure illustrates how both of the (recoupling) time domain periods can be processed (Fourier transformed) in order to obtain a dipolar or CSA-based 2D lineshape. Although information-rich, this $2 \mathrm{D}$ approach does take considerable time. In line with prior work, this paper (Mukhopadhyay et al., 2018) discussed and demonstrated an "accordion" approach in which multiple distinct recoupling periods are incremented in synchrony. This yields a faster " $1 \mathrm{D}$ " experiment that is must more time efficient than the $2 \mathrm{D}$ RACO spectra on uniformly labeled test compounds in Figure 9D. For more details, and the application to uniformly ${ }^{13} \mathrm{C},{ }^{15} \mathrm{~N}$-labeled proteins, the reader is referred to the original work (Mukhopadhyay et al., 2018).

\section{LONGER-RANGE VECTOR ANGLE MEASUREMENTS}

Most of the above examples are based on recoupling anisotropic interactions of neighboring (directly-bonded) atoms. However, useful angular constraints can also be derived from non-local interactions, involving atoms that are not directly bonded. Some CSA-CSA recoupling experiments fall into this category, but there are also dipolar based variants. This includes the recoupling of $\mathrm{N}-\mathrm{H}$ dipolar vectors for ${ }^{15} \mathrm{~N}$ nuclei in neighboring amino acids, in HNNH-type experiments (Reif et al., 2000; Rienstra et al., 2002a, Rienstra et al., 2002b; Franks et al., 2006, Franks et al., 2008). The utility of such vector angle (VEAN) constraints was nicely demonstrated in the high-resolution structure determination of the model protein GB1 (Franks et al., 2006, Franks et al., 2008; Wylie et al., 2011). The obtained N-H, N-H VEAN depends on several standard protein dihedral angles, as well as bond lengths and bond angles. To use these constraints, the authors directly incorporated the VEAN angle into the structure calculation routine, rather than attempt to back-calculate the individual $\phi / \psi / \chi$ angles. It is worth noting that these "remote" angle constraints can also take other forms, outside the $\mathrm{HNNH}$ variant, with any orientational constraint being potential valuable for structure determination. For example, the relative orientation (or projection angle $\theta$ ) between $(\mathrm{N}-\mathrm{H})_{\mathrm{i}+1}$ and $(\mathrm{Ca}-\mathrm{H})_{\mathrm{i}}$ have been used to constrain amyloid structure of a fragment of the transthyretin protein (Jaroniec et al., 2004).

\section{OTHER SSNMR PROBES OF DIHEDRAL ANGLES}

\section{Distance-Based Constraints}

Although not a focus of the current review, it is worth noting that many ssNMR distance measurements directly or indirectly constrain dihedral angles. In some cases, specific ssNMR experiments were designed with the explicit goal to determine dihedral angles via precise measurements of specific internuclear distances (Sinha and Hong, 2003; Wi and Spano, 2011; Hu et al., 2012; Pope et al., 2018). This includes for instance the so-called BARE (Backbone Recoupling) experiments that measure the distances between backbone nitrogens and carbonyls, with implications for the intervening backbone torsion angles ( $\mathrm{Hu}$ et al., 2012).

\section{Isotropic Chemical Shifts as Torsion Angle Constraints}

From the above it is clear that there is an impressive library of ssNMR torsion angle measurements, many of which were developed and demonstrated in the late 1990s and early 2000s. Nonentheless, since then many ssNMR protein structures were determined without use of these types of constraints. Instead, most structural ssNMR studies currently deploy isotropic chemical shifts to estimate the residue-specific backbone torsion angles. This is facilitated by the observation that backbone chemical shifts (along with the $\mathrm{C} \beta$ shift) are reliable indicators of local secondary structure, and can even be used for quantitative determinations of backbone dihedral angles (Cornilescu et al., 1999; Shen and Bax, 2007; Shen et al., 2009). Although developed for (and from) solution NMR protein structures, these algorithms have been shown to be similarly effective for ssNMR chemical shifts. These results have been sufficiently reliable that several papers deploying explicit torsion angle measurements note the high degree of consistency between the two approaches (Jaroniec et al., 2004; van der Wel et al., 2010). That said, both methods have their strengths and weaknesses, and can used as complementary tools (van der Wel et al., 2010; Hoop et al., 2016). For example, chemical shift-based estimates can be used to resolve ambiguities inherent in direct dihedral angle measurements (and vice versa).

\section{PROSPECTS FOR TORSION ANGLE APPLICATIONS}

These recent studies give a chance to consider the question of when and why to deploy torsion angle measurements. These experiments are in principle powerful, and they been used in recent years, but clearly not as widely as other structural ssNMR measurements. There are several reasons of this. One reason is that protein chemical shifts themselves give a lot of insight into the backbone torsion angles (i.e. secondary structure), with programs like TALOS allowing for a semiquantitative determination of the backbone torsion angles 
(Shen et al., 2009; van der Wel et al., 2010). Most recent ssNMR-based structures are based on combinations of distance measurements along with such chemical-shiftbased backbone angles. Although (typically) neither the distances nor the angles are extremely precise, their combination in sufficient numbers can yield good atomic structures of proteins (Loquet et al., 2008). Dihedral angle measurements may be used to improve the resolution of a structure derived from a combination of distance constraints and chemical shift information. This has been demonstrated for example in work by the Rienstra group on crystals of the model protein GB1 (Franks et al., 2008; Wylie et al., 2011). This enables higher resolution structures than otherwise accessible, but the question may arise whether the improvement in the structure quality warrants the amount of work (NMR time, but also data analysis). One possible approach would be to deploy these experiments in a targeted fashion: to enhance our understanding of especially important parts of protein structures, such as enzyme active sites, ion channel selectivity pores and similar (van der Wel et al., 2009; Caulkins et al., 2014; Wylie et al., 2014). Still, the implementation, execution, and interpretation of torsion angle measurements can be challenging, more so due to more limited prior experience with these methods in the overall ssNMR community (compared to canonical distance measurements). Thus, it may seem unclear when one would deploy dihedral angle measurements. We will examine some considerations or conditions that favor their use.

\section{Extensive Intermolecular Interactions}

Distance-based ssNMR structure determination borrows heavily from methods perfected in solution NMR structural biology. In dissolved or crystalline globular proteins, or in membrane-associated proteins, one can assume that most NMR-detected distance constraints reflect interactions within an individual protein (Loquet et al., 2008). However, this is not always a safe assumption, for example when studying amyloid fibrils in which the predominant residue-residue interactions may be inter- rather than intra-molecular. SSNMR studies of amyloid structures have addressed this by diluting labeled monomers in an excess of unlabeled monomers, prior to the assembly process. This approach allows for the suppression of intermolecular interactions among (labeled) atoms, thus revealing intramolecular interactions that define the monomer structure within the fibril. Unfortunately, it also implies a drastic loss of sensitivity, as the sample is now only partly filled with "visible" labeled monomers. Torsion angle measurements are designed to probe the very local environment of the (bond) angle of interest. This means that they deploy relatively modest dipolar recoupling times and are largely insensitive to the presence or absence of intermolecular interactions, and that they can always be used to probe the monomer structure even in fully labeled samples. Aside from the already mentioned signal-to-noise benefits, this may also be important for (biological) assemblies that are difficult or impossible to (re)assemble in vitro from monomers, and thus are either fully labeled or fully unlabeled.

\section{Assemblies With Inherent or Internal Polymorphism}

Another challenge faced in our recent studies of amyloid fibrils is that some fibrils contain the same monomer in two or three distinct configurations or conformations, as part of a complex or composite fiber architecture (van der Wel et al., 2010; Lewandowski et al., 2011; Hoop et al., 2016). This means that any single atom (or residue) yields multiple signals, which further complicates distance-based structural measurements (E.g., Figures 3, 4 show examples). Extensive signal overlap results that cannot be resolved by site-specific isotope labeling or spinsystem-based spectroscopic editing. Moreover, the effective signal to noise is decreased, as you are effectively studying a system that behaves as if it is twice or thrice as large (in terms of signal to noise). Yet, unlike a larger protein sequence, this challenge cannot be resolved by residue-specific labeling, truncation of the sequence, mutations, or segmental labeling. In such a case, distance measurements become less powerful, and torsion angle measurements can become an essential tool.

\section{Cases Where Chemical Shift Analysis Falls Short}

It was noted above that backbone dihedral angles can be probed via the chemical shift assignments of proteins, with the results sometimes being not much "worse" than much more timeconsuming torsion angle measurements (Figure 2F). However, even if the detection of extended $\beta$-strands and $\alpha$-helices is quite reliable, some non-standard motifs can be more challenging. In such cases chemical shift-based analysis alone may fail to resolve a reliable backbone conformation for a combination of observed shift values (see e.g., Figure 2J). Thus, it may be helpful to deploy targeted torsion angle measurements in such cases (Franks et al., 2008; van der Wel et al., 2010). Moreover, chemical shift analysis is limited to protein backbones, while ssNMR torsion angle measurements can be applied to side chains and non-protein biomolecules.

\section{Repetitive Sequences and Structures}

Our work on polyQ amyloid structure illustrates one interesting class of proteins where distance constraints fail to provide a complete answer. Highly repetitive sequences such as the polyQ proteins render distance constraints more difficult to obtain, or at least interpret. Biology features many repetitive protein sequences. Protein aggregation diseases often feature proteins with low complexity sequences, which are sometimes defined as prion-like sequence elements (Franzmann and Alberti, 2019). Besides several distinct polyQ disease proteins and highly sequence-repetitive prions, much interest extends also to the repetitive dipeptide-repeats associated with ALS disease (Odeh and Shorter, 2020; Schmitz et al., 2021). The aggregated and phase-separated states of these proteins remain as yet poorly understood, with studies by ssNMR likely being important to understanding their structure, dynamics and phase behavior. However, repetitive sequences go well beyond amyloidogenic proteins. A different example is collagen, which is an essential 
component of the extracellular matrix (ECM), where it helps define the structural characteristics of tissues. The (mechanical) properties of the ECM are of substantial biological interest, for instance in the context of cancer research and treatments (Venning et al., 2015). Already a topic of significant ssNMR studies (Goldberga et al., 2018), collagen's repetitive structure yields highly challenging spectra with highly overlapping signals, akin to the polyQ case study above. Dihedral angle measurements may be similarly useful for nonetheless probing the local structure in atomic detail. Similarly, the silk proteins produced by spiders and other animals are highly repetitive in sequence and have interesting structural properties that have been studied by ssNMR (van Beek and Meier, 2006; Holland et al., 2008). Also here dihedral angle measurements can be useful to probe their structures and structural transitions (van Beek and Meier, 2006).

\section{Applications Beyond Polypeptides}

Mostly we have focused on the study of protein samples. However, the use of dihedral angle measurements is also of interest for the study of other biomolecules (or non-biological samples). Besides repetitive protein structures, we may also consider applications to non-protein macromolecules of biological, biomedical and bioengineering interest. For instance (high-molecular weight) polysaccharides, RNA and DNA are increasingly studied by ssNMR, but present new challenges in terms of structure determination (Marchanka et al., 2015; El Hariri El Nokab and van der Wel, 2020).

However, also in small molecules (or short peptides), it can be difficult to gain sufficient information from distance constraints alone. In such a case, dihedral angle measurements can play an important role. In the context of peptides, this has now been well demonstrated (Rienstra et al., 2002b; Jaroniec et al., 2004; Bajaj et al., 2009; Barnes et al., 2009; van der Wel et al., 2010). Nice examples can also be found in the earlier literature, for example in studies of the retinal of the membrane protein rhodopsin (Feng et al., 1997b). The recent literature offers several other interesting case studies, as we have already seen above, in which torsion angle measurements probe the structure of small molecules bound to proteins, rather than the protein itself. This includes the example of a small molecule substrate bound to a receptor protein (Figure 5) (Edwards et al., 2010) and the amyloid-specific fluorescent dye congo red bound to HET-s fibrils (Gowda et al., 2017). It is likely that further applications like this can be expected in future work on, e.g., drug-protein, substrateenzyme and other such interactions.

\section{TECHNICAL CHALLENGES AND OPPORTUNITIES}

As with any (ssNMR) experiment, the torsion angle measurements offer both unique strengths and also specific challenges. This section notes a few specific challenges, but follows this with perspectives on how they can be overcome (partly with the help of modern MAS ssNMR equipment). Like with other structural ssNMR studies reliant on anisotropic interactions, any dynamics in the molecular system can interfere with the execution and/or analysis of torsion angles. Since dynamics modulate both dipolar interactions and CSA tensors, this would naturally cause problems. These dynamics can be important and relevant, since biological contexts often require dynamics (e.g., in enzymes or ion channels) to achieve proper function. On the one hand, it may be possible to account for certain types of (anisotropic) motion in the data analysis, as exemplified in prior studies that characterized such motion by ssNMR (Hu et al., 2010; Li and Hong, 2011). Another workaround could be the use of low-temperature experiments, which are increasingly accessible with the enhanced availability of low-temperature dynamic nuclear polarization (DNP) equipment (Lilly Thankamony et al., 2017), and can permit the execution of dihedral angle measurements at reduced temperatures where molecular motion is suppressed (Bajaj et al., 2009).

One challenge independent of motion is the inherently low sensitivity of the experiments. Whilst applications to crystalline peptides have been quite effective and convincing, applications to more complex systems are fairly demanding. As already discussed above, a combination of factors contribute to this challenge. On the one hand, the nature of the pulse sequences is such the signals are purposely decreased, and we monitor the differential degree of dephasing to distinguish the differences in structure. Crucially, for several types of measurements certain ranges of angles are close together in terms of their dephasing curves. This places significant demands on the signal to noise. The good news is the techniques and equipment available for MAS ssNMR have greatly improved since the dihedral angle measurements were first developed. On the one hand, we already discussed DNP for its low temperature capabilities. This technique also offers substantial signal enhancements, which even permit applications to natural abundance proteins and materials within reach. Notably, recent DNP studies of unlabeled proteins and other organic (bio)molecules (Märker et al., 2017; Smith et al., 2018) already depend heavily on the kinds of DQ experiments at the heart of several types of dihedral angle measurements, as reviewed above.

In addition to DNP technology, we also see the gains in the application of high field ssNMR and especially also $\left({ }^{1} \mathrm{H}\right.$-detected $)$ fast MAS $(>60 \mathrm{kHz})$. Most of the example data discussed in this review article were obtained with MAS rates between 10 and $15 \mathrm{kHz}$ (reflecting typically 3.2 and $4 \mathrm{~mm}$ MAS rotor diameters). High MAS rates that exceed even $100 \mathrm{kHz}$ are now available, permitting ${ }^{1} \mathrm{H}$ detection and other new pulse sequences (BarbetMassin et al., 2014; Zhang et al., 2017; Ji et al., 2021). This can enable new types of experiments and thereby new structural insights, often with improved sensitivity and time-savings. However at the same time, some traditional pulse sequence elements become difficult or impossible to implement, for instance due to the overly short rotor period lengths or the high RF power requirements. This for instance means that REDOR-based methods may not work, requiring the deployment of new schemes. This has opened up new developments of various sorts of MAS ssNMR experiments, such as tailored assignment and relaxation measurements (Barbet-Massin et al., 2014; Zhang et al., 2017; Ji et al., 2021), 
but the development of (new) dihedral angle experiments has lagged behind (Hou et al., 2010).

A final practical challenge relates to the implementation and analysis of torsion angle experiments. Fewer groups have handson experience with dihedral angle experiments, compared to the more widely used distance measurements. Moreover, the configuration and implementation of these experiments is arguably more involved than, e.g., a traditional DARR-based distance measurement. Also the interpretation of the obtained results is perhaps less intuitive than looking for the presence or absence of cross peaks in typical distance measurements. One helpful development is the availability of various efficient and flexible numerical simulation packages, which can facilitate both test simulations to better understand the use of these experiments, and can also enable efficient analysis and interpretation of obtained results (Bak et al., 2000; Veshtort and Griffin, 2006).

With all these technical and hardware enhancements, it seems likely that many new and useful dihedral angle measurements may be introduced in the future. And it also seems plausible that existing techniques may find wider adoption and application to suitable systems, whether biological or non-biological in nature.

\section{DISCUSSION}

In this article we have examined several recent applications and methodologies of ssNMR dihedral angle measurements, focusing on those techniques based on the recoupling of anisotropic dipolar and/or CSA tensors. With the advent of highly productive distance and shift-based structure determination techniques for ssNMR-based structural biology, these direct dihedral angle measurements have been a bit left outside the mainstream. Yet, we have seen how they can be powerful and essential for tackling various biologically important questions, ranging from amyloid fiber structure

\section{REFERENCES}

Armstrong, N., and Gouaux, E. (2000). Mechanisms for Activation and Antagonism of an AMPA-Sensitive Glutamate Receptor. Neuron 28, 165-181. doi:10.1016/S0896-6273(00)00094-5

Bajaj, V. S., van der Wel, P. C. A., and Griffin, R. G. (2009). Observation of a LowTemperature, Dynamically Driven Structural Transition in a Polypeptide by SolidState NMR Spectroscopy. J. Am. Chem. Soc. 131, 118-128. doi:10.1021/ja8045926

Bak, M., Rasmussen, J. T., and Nielsen, N. C. (2000). SIMPSON: a General Simulation Program for Solid-State NMR Spectroscopy. J. Magn. Reson. 147, 296-330. doi:10.1006/jmre.2000.2179

Barbet-Massin, E., Pell, A. J., Retel, J. S., Andreas, L. B., Jaudzems, K., Franks, W. T., et al. (2014). Rapid Proton-Detected NMR Assignment for Proteins with Fast Magic Angle Spinning. J. Am. Chem. Soc. 136, 12489-12497. doi:10.1021/ja507382j

Barnes, A. B., Andreas, L. B., Huber, M., Ramachandran, R., van der Wel, P. C. A., Veshtort, M., et al. (2009). High-resolution Solid-State NMR Structure of AlanylProlyl-glycine. J. Magn. Reson. 200, 95-100. doi:10.1016/j.jmr.2009.06.009

Bayro, M. J., Huber, M., Ramachandran, R., Davenport, T. C., Meier, B. H., Ernst, M., et al. (2009). Dipolar Truncation in Magic-Angle Spinning NMR Recoupling Experiments. J. Chem. Phys. 130, 114506. doi:10.1063/1.3089370

Bower, P. V., Oyler, N., Mehta, M. A., Long, J. R., Stayton, P. S., and Drobny, G. P. (1999). Determination of Torsion Angles in Proteins and Peptides Using Solid State NMR. J. Am. Chem. Soc. 121, 8373-8375. doi:10.1021/ja991330q determination to receptor-substrate interactions. It is furthermore anticipated that there is significant room for enhancing the utility of these techniques, as future studies will surely integrate these techniques with modern MAS ssNMR techniques such as ${ }^{1} \mathrm{H}$-detected fast MAS and DNP. Thus, we foresee an expansion of the role of these methods toward broader application in the ssNMR community, with valuable roles in studies of biological and non-biological systems, both with and without stable-isotope labeling.

\section{AUTHOR CONTRIBUTIONS}

PvdW wrote and edited the article.

\section{FUNDING}

Our studies of polyglutamine amyloid structure have been funded by the NIH NIGMS R01 GM112678 (United States) and the Campagne Team Huntington foundation in Netherlands.

\section{ACKNOWLEDGMENTS}

The author thanks members of the Van der Wel group for their contributions to the discussed work and comments on the article draft. I also thank Matthias Ernst for kindly providing the data plotted in Figure 8C. Molecular graphics were made with UCSF ChimeraX, developed by the Resource for Biocomputing, Visualization, and Informatics at the University of California, San Francisco, with support from National Institutes of Health R01-GM129325 and the Office of Cyber Infrastructure and Computational Biology, National Institute of Allergy and Infectious Diseases.

Caulkins, B. G., Bastin, B., Yang, C., Neubauer, T. J., Young, R. P., Hilario, E., et al. (2014). Protonation States of the Tryptophan Synthase Internal Aldimine Active Site from Solid-State NMR Spectroscopy: Direct Observation of the Protonated Schiff Base Linkage to Pyridoxal-5'-Phosphate. J. Am. Chem. Soc. 136, 12824-12827. doi:10.1021/ja506267d

Chan, J. C. C., and Tycko, R. (2003). Solid-State NMR Spectroscopy Method for Determination of the Backbone Torsion Angle $\psi$ in Peptides with Isolated Uniformly Labeled Residues. J. Am. Chem. Soc. 125, 11828-11829. doi:10.1021/ja0369820

Cornilescu, G., Delaglio, F., and Bax, A. (1999). Protein Backbone Angle Restraints from Searching a Database for Chemical Shift and Sequence Homology. J. Biomol. NMR 13, 289-302. doi:10.1023/a:1008392405740

Costa, P. R., Gross, J. D., Hong, M., and Griffin, R. G. (1997). Solid-state NMR Measurement of $\Psi$ in Peptides: a NCCN 2Q-Heteronuclear Local Field experiment. Chem. Phys. Lett. 280, 95-103. doi:10.1016/s0009-2614(97)01107-x

Dunbrack, R. L. (2002). Rotamer Libraries in the 21st century. Curr. Opin. Struct. Biol. 12, 431-440. doi:10.1016/s0959-440x(02)00344-5

Edwards, R., Madine, J., Fielding, L., and Middleton, D. A. (2010). Measurement of Multiple Torsional Angles from One-Dimensional Solid-State NMR Spectra: Application to the Conformational Analysis of a Ligand in its Biological Receptor Site. Phys. Chem. Chem. Phys. 12, 13999-14008. doi:10.1039/c0cp00326c

El Hariri El Nokab, M., and van der Wel, P. C. A. (2020). Use of Solid-State NMR Spectroscopy for Investigating Polysaccharide-Based Hydrogels: A Review. Carbohydr. Polym. 240, 116276. doi:10.1016/j.carbpol.2020.116276 
Feng, X., Edén, M., Brinkmann, A., Luthman, H., Eriksson, L., Gräslund, A., et al. (1997a). Direct Determination of a Peptide Torsional Angle $\psi$ by Double-Quantum Solid-State NMR. J. Am. Chem. Soc. 119, 12006-12007. doi:10.1021/ja972252e

Feng, X., Lee, Y. K., Sandström, D., Edén, M., Maisel, H., Sebald, A., et al. (1996). Direct Determination of a Molecular Torsional Angle by Solid-State NMR. Chem. Phys. Lett. 257, 314-320. doi:10.1016/0009-2614(96)00558-1

Feng, X., Verdegem, P. J. E., Lee, Y. K., Sandström, D., Edén, M., Bovee-Geurts, P., et al. (1997b). Direct Determination of a Molecular Torsional Angle in the Membrane Protein Rhodopsin by Solid-State NMR. J. Am. Chem. Soc. 119, 6853-6857. doi:10.1021/ja970710d

Franks, W. T., Wylie, B. J., Schmidt, H. L. F., Nieuwkoop, A. J., Mayrhofer, R.-M., Shah, G. J., et al. (2008). Dipole Tensor-Based Atomic-Resolution Structure Determination of a Nanocrystalline Protein by Solid-State NMR. Proc. Natl. Acad. Sci. 105, 4621-4626. doi:10.1073/pnas.0712393105

Franks, W. T., Wylie, B. J., Stellfox, S. A., and Rienstra, C. M. (2006). Backbone Conformational Constraints in a Microcrystalline U-15n-Labeled Protein by 3D Dipolar-Shift Solid-State NMR Spectroscopy. J. Am. Chem. Soc. 128, 3154-3155. doi:10.1021/ja058292x

Franzmann, T. M., and Alberti, S. (2019). Prion-like Low-Complexity Sequences: Key Regulators of Protein Solubility and Phase Behavior. J. Biol. Chem. 294, 7128-7136. doi:10.1074/jbc.TM118.001190

Fujiwara, T., Shimomura, T., and Akutsu, H. (1997). Multidimensional Solid-State Nuclear Magnetic Resonance for Correlating Anisotropic Interactions under Magic-Angle Spinning Conditions. J. Magn. Reson. 124, 147-153. doi:10.1006/jmre.1996.1022

Goldberga, I., Li, R., and Duer, M. J. (2018). Collagen Structure-Function Relationships from Solid-State NMR Spectroscopy. Acc. Chem. Res. 51, 1621-1629. doi:10.1021/acs.accounts.8b00092

Gowda, C., Zandomeneghi, G., Zimmermann, H., Schütz, A. K., Böckmann, A., Ernst, M., et al. (2017). The Conformation of the Congo-red Ligand Bound to Amyloid Fibrils HET-S(218-289): a Solid-State NMR Study. J. Biomol. NMR 69, 207-213. doi:10.1007/s10858-017-0148-Z

Hintze, B. J., Lewis, S. M., Richardson, J. S., and Richardson, D. C. (2016). Molprobity's Ultimate Rotamer-Library Distributions for Model Validation. Proteins 84, 1177-1189. doi:10.1002/prot.25039

Holland, G. P., Creager, M. S., Jenkins, J. E., Lewis, R. V., and Yarger, J. L. (2008). Determining Secondary Structure in Spider Dragline Silk by Carbon-Carbon Correlation Solid-State NMR Spectroscopy. J. Am. Chem. Soc. 130, 9871-9877. doi:10.1021/ja8021208

Hong, M. (2006). “Torsion Angle Determination by Solid-State NMR,” in Modern Magnetic Resonance (Dordrecht: Springer), 723-729.

Hong, M. (1999). Determination of Multiple $\varphi$-Torsion Angles in Proteins by Selective and Extensive 13C Labeling and Two-Dimensional Solid-State NMR. J. Magn. Reson. 139, 389-401. doi:10.1006/jmre.1999.1805

Hong, M., Gross, J. D., and Griffin, R. G. (1997a). Site-Resolved Determination of Peptide Torsion Angle $\varphi$ from the Relative Orientations of Backbone N-H and C-H Bonds by Solid-State NMR. J. Phys. Chem. B 101, 5869-5874. doi:10.1021/jp970887u

Hong, M., Gross, J. D., Hu, W., and Griffin, R. G. (1998). Determination of the Peptide Torsion Angle $\varphi$ by $15 \mathrm{~N}$ Chemical Shift and13Ca-1HaDipolar Tensor Correlation in Solid-State MAS NMR. J. Magn. Reson. 135, 169-177. doi:10.1006/jmre.1998.1573

Hong, M., Gross, J. D., Rienstra, C. M., Griffin, R. G., Kumashiro, K. K., and Schmidt-Rohr, K. (1997b). Coupling Amplification in 2D MAS NMR and its Application to Torsion Angle Determination in Peptides. J. Magn. Reson. 129, 85-92. doi:10.1006/jmre.1997.1242

Hong, M., and Wi, S. (2006). "Torsion Angle Determination in Biological Solids by Solid-State Nuclear Magnetic Resonance," in NMR Spectroscopy of Biological Solids. Editor A. Ramamoorthy (Boca Raton, FL: CRC Press), 88-122.

Hoop, C. L., Lin, H.-K., Kar, K., Hou, Z., Poirier, M. A., Wetzel, R., et al. (2014). Polyglutamine Amyloid Core Boundaries and Flanking Domain Dynamics in Huntingtin Fragment Fibrils Determined by Solid-State Nuclear Magnetic Resonance. Biochemistry 53, 6653-6666. doi:10.1021/bi501010q

Hoop, C. L., Lin, H.-K., Kar, K., Magyarfalvi, G., Lamley, J. M., Boatz, J. C., et al. (2016). Huntingtin Exon 1 Fibrils Feature an Interdigitated $\beta$-hairpin-based Polyglutamine Core. Proc. Natl. Acad. Sci. USA 113, 1546-1551. doi:10.1073/pnas.1521933113

Hou, G., Paramasivam, S., Byeon, I.-J. L., Gronenborn, A. M., and Polenova, T. (2010). Determination of Relative Tensor Orientations by $\gamma$-encoded Chemical Shift Anisotropy/heteronuclear Dipolar Coupling 3D NMR Spectroscopy in Biological Solids. Phys. Chem. Chem. Phys. 12, 14873-14883. doi:10.1039/ c0cp00795a
Hovmöller, S., Zhou, T., and Ohlson, T. (2002). Conformations of Amino Acids in Proteins. Acta Crystallogr. D Biol. Cryst. 58, 768-776. doi:10.1107/S0907444902003359

Hu, F., Luo, W., and Hong, M. (2010). Mechanisms of Proton Conduction and Gating in Influenza M2 Proton Channels from Solid-State NMR. Science 330, 505-508. doi:10.1126/science.1191714

Hu, K.-N., Qiang, W., Bermejo, G. A., Schwieters, C. D., and Tycko, R. (2012). Restraints on Backbone Conformations in Solid State NMR Studies of Uniformly Labeled Proteins from Quantitative Amide $15 \mathrm{~N}-15 \mathrm{~N}$ and Carbonyl 13C-13C Dipolar Recoupling Data. J. Magn. Reson. 218, 115-127. doi:10.1016/j.jmr.2012.03.001

Isas, J. M., Langen, R., and Siemer, A. B. (2015). Solid-State Nuclear Magnetic Resonance on the Static and Dynamic Domains of Huntingtin Exon-1 Fibrils. Biochemistry 54, 3942-3949. doi:10.1021/acs.biochem.5b00281

Ishii, Y., Terao, T., and Kainosho, M. (1996). Relayed Anisotropy Correlation NMR: Determination of Dihedral Angles in Solids. Chem. Phys. Lett. 256, 133-140. doi:10.1016/0009-2614(96)00426-5

IUPAC-IUB Commission on Biochemical Nomenclature (1970). IUPAC-IUB Commission on Biochemical Nomenclature. Abbreviations and Symbols for the Description of the Conformation of Polypeptide Chains. J. Mol. Biol. 52, 1-17. doi:10.1016/0022-2836(70)90173-7

Jaroniec, C. P., MacPhee, C. E., Bajaj, V. S., McMahon, M. T., Dobson, C. M., and Griffin, R. G. (2004). High-resolution Molecular Structure of a Peptide in an Amyloid Fibril Determined by Magic Angle Spinning NMR Spectroscopy. Proc. Natl. Acad. Sci. 101, 711-716. doi:10.1073/pnas.0304849101

Ji, Y., Liang, L., Bao, X., and Hou, G. (2021). Recent Progress in Dipolar Recoupling Techniques under Fast MAS in Solid-State NMR Spectroscopy. Solid State. Nucl. Magn. Reson. 112, 101711. doi:10.1016/j.ssnmr.2020.101711

Ladizhansky, V. (2009). "Dipolar-Based Torsion Angle Measurements for Protein Structure Determination," in Encyclopedia of Magnetic Resonance. Editors R. K. Harris and R. Wasylishen (Chichester, United Kingdom: John Wiley). doi:10.1002/9780470034590.emrstm1153

Ladizhansky, V., Jaroniec, C. P., Diehl, A., Oschkinat, H., and Griffin, R. G. (2003). Measurement of Multiple $\psi$ Torsion Angles in Uniformly 13C,15N-Labeled a-Spectrin SH3 Domain Using 3D 15N-13C-13C-15N MAS DipolarChemical Shift Correlation Spectroscopy. J. Am. Chem. Soc. 125, 6827-6833. doi: $10.1021 /$ ja029082c

Ladizhansky, V., Veshtort, M., and Griffin, R. G. (2002). NMR Determination of the Torsion Angle $\Psi$ in $\alpha$-Helical Peptides and Proteins: The HCCN Dipolar Correlation Experiment. J. Magn. Reson. 154, 317-324. doi:10.1006/jmre.2001.2488

Lewandowski, J. R., van der Wel, P. C. A., Rigney, M., Grigorieff, N., and Griffin, R. G. (2011). Structural Complexity of a Composite Amyloid Fibril. J. Am. Chem. Soc. 133, 14686-14698. doi:10.1021/ja203736z

Li, S., and Hong, M. (2011). Protonation, Tautomerization, and Rotameric Structure of Histidine: a Comprehensive Study by Magic-Angle-Spinning Solid-State NMR. J. Am. Chem. Soc. 133, 1534-1544. doi:10.1021/ja108943n

Lilly Thankamony, A. S., Kaushik, M., Corzilius, B., and Corzilius, B. (2017). Dynamic Nuclear Polarization for Sensitivity Enhancement in Modern Solid-State NMR. Prog. Nucl. Magn. Reson. Spectrosc. 102-103, 120-195. doi:10.1016/j.pnmrs.2017.06.002

Loquet, A., Bardiaux, B., Gardiennet, C., Blanchet, C., Baldus, M., Nilges, M., et al. (2008). 3D Structure Determination of the Crh Protein from Highly Ambiguous Solid-State NMR Restraints. J. Am. Chem. Soc. 130, 3579-3589. doi:10.1021/ja078014t

Lovell, S. C., Word, J. M., Richardson, J. S., and Richardson, D. C. (2000). The Penultimate Rotamer Library. Proteins 40, 389-408. doi:10.1002/10970134(20000815)40:3<389:aid-prot50>3.0.co;2-2

Marchanka, A., Simon, B., Althoff-Ospelt, G., and Carlomagno, T. (2015). RNA Structure Determination by Solid-State NMR Spectroscopy. Nat. Commun. 6 , 7024. doi:10.1038/ncomms8024

Märker, K., Paul, S., Fernández-de-Alba, C., Lee, D., Mouesca, J.-M., Hediger, S., et al. (2017). Welcoming Natural Isotopic Abundance in Solid-State NMR: Probing $\pi$-stacking and Supramolecular Structure of Organic Nanoassemblies Using DNP. Chem. Sci. 8, 974-987. doi:10.1039/C6SC02709A

Markley, J. L., Bax, A., Arata, Y., Hilbers, C. W., Kaptein, R., Sykes, B. D., et al. (1998). Recommendations for the Presentation of NMR Structures of Proteins and Nucleic Acids (IUPAC Recommendations 1998). Pure Appl. Chem. 70, 117-142. doi:10.1351/pac199870010117

Matlahov, I., and van der Wel, P. C. (2019). Conformational Studies of Pathogenic Expanded Polyglutamine Protein Deposits from Huntington's Disease. Exp. Biol. Med. (Maywood) 244, 1584-1595. doi:10.1177/1535370219856620 
Mukhopadhyay, D., Gupta, C., Theint, T., and Jaroniec, C. P. (2018). Peptide Bond Conformation in Peptides and Proteins Probed by Dipolar Coupling-Chemical Shift Tensor Correlation Solid-State NMR. J. Magn. Reson. 297, 152-160. doi:10.1016/j.jmr.2018.10.015

Musacchio, A., Noble, M., Pauptit, R., Wierenga, R., and Saraste, M. (1992). Crystal Structure of a Src-Homology 3 (SH3) Domain. Nature 359, 851-855. doi:10.1038/359851a0

Nelson, R., Sawaya, M. R., Balbirnie, M., Madsen, A. Ø., Riekel, C., Grothe, R., et al. (2005). Structure of the Cross- $\beta$ Spine of Amyloid-like Fibrils. Nature 435, 773-778. doi:10.1038/nature03680

Odeh, H. M., and Shorter, J. (2020). Arginine-rich Dipeptide-Repeat Proteins as Phase Disruptors in C9-ALS/FTD. Emerging Top. Life Sci. 4, 293-305. doi:10.1042/ETLS20190167

Pettersen, E. F., Goddard, T. D., Huang, C. C., Meng, E. C., Couch, G. S., Croll, T. I., et al. (2021). UCSF ChimeraX : Structure Visualization for Researchers, Educators, and Developers. Protein Sci. 30, 70-82. doi:10.1002/pro.3943

Pope, G. M., Hung, I., Gan, Z., Mobarak, H., Widmalm, G., and Harper, J. K. (2018). Exploiting 13C/14N Solid-State NMR Distance Measurements to Assign Dihedral Angles and Locate Neighboring Molecules. Chem. Commun. 54, 6376-6379. doi:10.1039/c8cc02597e

Ramachandran, G. N., and Sasisekharan, V. (1968). "Conformation of Polypeptides and Proteins," in Advances in Protein Chemistry (Elsevier), 283-437. doi:10.1016/S0065-3233(08)60402-7

Reif, B., Hohwy, M., Jaroniec, C. P., Rienstra, C. M., and Griffin, R. G. (2000). NHNH Vector Correlation in Peptides by Solid-State NMR. J. Magn. Reson. 145, 132-141. doi:10.1006/jmre.2000.2067

Rienstra, C. M., Hohwy, M., Mueller, L. J., Jaroniec, C. P., Reif, B., and Griffin, R. G. (2002a). Determination of Multiple Torsion-Angle Constraints in U-13C,15NLabeled Peptides: 3D 1H-15N-13C-1H Dipolar Chemical Shift NMR Spectroscopy in Rotating Solids. J. Am. Chem. Soc. 124, 11908-11922. doi:10.1021/ja020802p

Rienstra, C. M., Tucker-Kellogg, L., Jaroniec, C. P., Hohwy, M., Reif, B., McMahon, M. T., et al. (2002b). De Novo determination of Peptide Structure with SolidState Magic-Angle Spinning NMR Spectroscopy. Proc. Natl. Acad. Sci. 99, 10260-10265. doi:10.1073/pnas.152346599

Sawaya, M. R., Hughes, M. P., Rodriguez, J. A., Riek, R., and Eisenberg, D. S. (2021). The Expanding Amyloid Family: Structure, Stability, Function, and Pathogenesis. Cell 184, 4857-4873. doi:10.1016/j.cell.2021.08.013

Schmitz, A., Pinheiro Marques, J., Oertig, I., Maharjan, N., and Saxena, S. (2021). Emerging Perspectives on Dipeptide Repeat Proteins in C9ORF72 ALS/FTD. Front. Cel. Neurosci. 15, 637548. doi:10.3389/fncel.2021.637548

Schneider, R., Schumacher, M. C., Mueller, H., Nand, D., Klaukien, V., Heise, H., et al. (2011). Structural Characterization of Polyglutamine Fibrils by Solid-State NMR Spectroscopy. J. Mol. Biol. 412, 121-136. doi:10.1016/ j.jmb.2011.06.045

Schütz, A. K., Soragni, A., Hornemann, S., Aguzzi, A., Ernst, M., Böckmann, A., et al. (2011). The Amyloid-Congo Red Interface at Atomic Resolution. Angew. Chem. Int. Ed. 50, 5956-5960. doi:10.1002/anie.201008276

Sharma, D., Shinchuk, L. M., Inouye, H., Wetzel, R., and Kirschner, D. A. (2005). Polyglutamine Homopolymers Having 8-45 Residues Form Slablike $\beta$-crystallite Assemblies. Proteins 61, 398-411. doi:10.1002/prot.20602

Shen, Y., and Bax, A. (2007). Protein Backbone Chemical Shifts Predicted from Searching a Database for Torsion Angle and Sequence Homology. J. Biomol. NMR 38, 289-302. doi:10.1007/s10858-007-9166-6

Shen, Y., Delaglio, F., Cornilescu, G., and Bax, A. (2009). TALOS+: a Hybrid Method for Predicting Protein Backbone Torsion Angles from NMR Chemical Shifts. J. Biomol. NMR 44, 213-223. doi:10.1007/s10858-009-9333-Z

Sinha, N., and Hong, M. (2003). X-1H Rotational-echo Double-Resonance NMR for Torsion Angle Determination of Peptides. Chem. Phys. Lett. 380, 742-748. doi:10.1016/j.cplett.2003.09.088

Sivanandam, V. N., Jayaraman, M., Hoop, C. L., Kodali, R., Wetzel, R., and van der Wel, P. C. A. (2011). The Aggregation-Enhancing Huntingtin N-Terminus Is Helical in Amyloid Fibrils. J. Am. Chem. Soc. 133, 4558-4566. doi:10.1021/ ja110715f

Smith, A. N., Märker, K., Piretra, T., Boatz, J. C., Matlahov, I., Kodali, R., et al. (2018). Structural Fingerprinting of Protein Aggregates by Dynamic Nuclear Polarization-Enhanced Solid-State NMR at Natural Isotopic Abundance. J. Am. Chem. Soc. 140, 14576-14580. doi:10.1021/jacs.8b09002
Torbeev, V. Y., and Hilvert, D. (2013). Both the Cis-Trans Equilibrium and Isomerization Dynamics of a Single Proline Amide Modulate 2microglobulin Amyloid Assembly. Proc. Natl. Acad. Sci. 110, 20051-20056. doi:10.1073/pnas.1310414110

van Beek, J. D., and Meier, B. H. (2006). A DOQSY Approach for the Elucidation of Torsion Angle Distributions in Biopolymers: Application to Silk. J. Magn. Reson. 178, 106-120. doi:10.1016/j.jmr.2005.09.004

van der Wel, P. C. A., Eddy, M. T., Ramachandran, R., and Griffin, R. G. (2009). Targeted13C-13C Distance Measurements in a Microcrystalline Protein via J-Decoupled Rotational Resonance Width Measurements. ChemPhysChem 10, 1656-1663. doi:10.1002/cphc.200900102

van der Wel, P. C. A. (2017). Insights into Protein Misfolding and Aggregation Enabled by Solid-State NMR Spectroscopy. Solid State. Nucl. Magn. Reson. 88, 1-14. doi:10.1016/j.ssnmr.2017.10.001

van der Wel, P. C. A., Lewandowski, J. R., and Griffin, R. G. (2007). Solid-state NMR Study of Amyloid Nanocrystals and Fibrils Formed by the Peptide GNNQQNY from Yeast Prion Protein Sup35p. J. Am. Chem. Soc. 129, 5117-5130. doi:10.1021/ja068633m

van der Wel, P. C. A., Lewandowski, J. R., and Griffin, R. G. (2010). Structural Characterization of GNNQQNY Amyloid Fibrils by Magic Angle Spinning NMR. Biochemistry 49, 9457-9469. doi:10.1021/bi100077x

Van der Wel, P. C. A. (2018). New Applications of Solid-State NMR in Structural Biology. Emerg. Top. Life Sci. 2, 57-67. doi:10.1042/ETLS20170088

Venning, F. A., Wullkopf, L., and Erler, J. T. (2015). Targeting ECM Disrupts Cancer Progression. Front. Oncol. 5, 1377. doi:10.3389/fonc.2015.00224

Veshtort, M., and Griffin, R. G. (2006). SPINEVOLUTION: a Powerful Tool for the Simulation of Solid and Liquid State NMR Experiments. J. Magn. Reson. 178, 248-282. doi:10.1016/j.jmr.2005.07.018

Weliky, D. P., and Tycko, R. (1996). Determination of Peptide Conformations by Two-Dimensional Magic Angle Spinning NMR Exchange Spectroscopy with Rotor Synchronization. J. Am. Chem. Soc. 118, 8487-8488. doi:10.1021/ ja960178e

Wetzel, R. (2012). Physical Chemistry of Polyglutamine: Intriguing Tales of a Monotonous Sequence. J. Mol. Biol. 421, 466-490. doi:10.1016/ j.jmb.2012.01.030

Wi, S., and Spano, J. (2011). Site-specific $\phi$ - and $\psi$-torsion Angle Determination in a Uniformly/extensively 13C- and 15N-Labeled Peptide. J. Magn. Reson. 212, 431-439. doi:10.1016/j.jmr.2011.08.019

Wylie, B. J., Bhate, M. P., and McDermott, A. E. (2014). Transmembrane Allosteric Coupling of the gates in a Potassium Channel. Proc. Natl. Acad. Sci. USA 111, 185-190. doi:10.1073/pnas.1319577110

Wylie, B. J., Sperling, L. J., Nieuwkoop, A. J., Franks, W. T., Oldfield, E., and Rienstra, C. M. (2011). Ultrahigh Resolution Protein Structures Using NMR Chemical Shift Tensors. Proc. Natl. Acad. Sci. 108, 16974-16979. doi:10.1073/ pnas. 1103728108

Yakupova, E. I., Bobyleva, L. G., Vikhlyantsev, I. M., and Bobylev, A. G. (2019). Congo Red and Amyloids: History and Relationship. Biosci. Rep. 39, BSR20181415. doi:10.1042/BSR20181415

Zhang, R., Mroue, K. H., and Ramamoorthy, A. (2017). Proton-Based Ultrafast Magic Angle Spinning Solid-State NMR Spectroscopy. Acc. Chem. Res. 50, 1105-1113. doi:10.1021/acs.accounts.7b00082

Conflict of Interest: The authors declare that the research was conducted in the absence of any commercial or financial relationships that could be construed as a potential conflict of interest.

Publisher's Note: All claims expressed in this article are solely those of the authors and do not necessarily represent those of their affiliated organizations, or those of the publisher, the editors and the reviewers. Any product that may be evaluated in this article, or claim that may be made by its manufacturer, is not guaranteed or endorsed by the publisher.

Copyright $\odot 2021$ van der Wel. This is an open-access article distributed under the terms of the Creative Commons Attribution License (CC BY). The use, distribution or reproduction in other forums is permitted, provided the original author $(s)$ and the copyright owner(s) are credited and that the original publication in this journal is cited, in accordance with accepted academic practice. No use, distribution or reproduction is permitted which does not comply with these terms. 